\title{
A Community Related Influence Evaluation Model for Social Networks with a Dynamic Perspective
}

\author{
Meizi Li, ${ }^{1,2}$ Yang Xiang, ${ }^{1}$ Qian Zhang, ${ }^{2}$ and Bo Zhang $\mathbb{D}^{2}$ \\ ${ }^{1}$ College of Electronics and Information Engineering, Tongji University, Shanghai 201804, China \\ ${ }^{2}$ College of Information, Mechanical and Electrical Engineering, Shanghai Normal University, Shanghai 200234, China \\ Correspondence should be addressed to Bo Zhang; zhangbo@shnu.edu.cn
}

Received 3 November 2017; Revised 16 May 2018; Accepted 17 June 2018; Published 11 July 2018

Academic Editor: Emilio Insfran Pelozo

Copyright (c) 2018 Meizi Li et al. This is an open access article distributed under the Creative Commons Attribution License, which permits unrestricted use, distribution, and reproduction in any medium, provided the original work is properly cited.

\begin{abstract}
The nature of free information diffusion and sharing in social networks (SNs) has increasingly attracted users to gather in communities to facilitate communication, resulting in increased challenges to community influence analysis in SNs. In SNs, the ability to evaluate how a community affects other users and communities is essential for further applications. Although considerable effort has been expended on the influence evaluation of individuals, little attention has been paid to influence evaluation of the community as a whole-particularly when considering the inherent features of dynamic changes of influence over time. This paper proposes a community related influence evaluation model with a dynamic perspective (D-CIEM) that reflects two types of influence, entity influence and community-initiated influence, by considering dynamic effects over time. Our work includes the following main points: first, we address the framework of D-CIEM and its related formal definitions; then, we propose dynamic entity influence evaluation in D-CIEM, i.e., user influence and community influence evaluation with dynamic factors. We present community-initiated dynamic influence evaluation methods, including methods to calculate community influence for internal and external users and for other communities. Finally, we provide evaluation results to verify the performance of our model.
\end{abstract}

\section{Introduction}

Social networks have garnered increasing attention for both personal communications and business transactions $[1,2]$. A social network (SN) is composed of numerous individual users who join the social network and impact each other to various degrees through the platform [3]. In contrast to other traditional web platforms, such as BBS and user forums, users in SNs have more freedom to join entity-oriented groups, called "communities" in SNs, to facilitate their daily communications and interactions. As an organization with massive numbers of members, a community naturally has a wider impact on users than does a single individual in an SN [4]. Therefore, it is important to analyze the potential influence of the $\mathrm{SN}$ community as an entity. However, communities in SNs have characteristics such as dynamic member organization and complex inner relationships between members that create challenges to analysis.

The problem of influence evaluation is of wide concern $[2,4-8]$. By measuring the properties of an entity in a social network, it is possible to determine how much influence that entity has and how it impacts the social network; therefore, influence analysis is an important facet of social network analysis [8]. Most studies that evaluate individual influence are based on users' personal properties, such as their profile attributes or on their connected relationships and interaction records $[9,10]$. However, little attention has been paid to mining another significant entity in SNs, i.e., community influence. Which perspectives are considered is critical for community influence analysis. Essentially, the influences of communities on users are the results of user interactions: users have strong influence on their friends, and respected experts strongly influence their fans. Therefore, the influence of a community can be evaluated by factors relating to that community's interaction with other entities (including both users and other communities in $\mathrm{SNs}$ ).

Some efforts have recently been made to evaluate influence in social networks [11]. There are two kinds of influence evaluation methods in social networks: single individual influence evaluation $[1,3,5,6,9,10,12-15]$ and 
group-organized influence evaluation $[4,7,8]$. The former focuses on measuring the impact of a single user based on that user's properties, while the latter shows how members use their influence to establish community influence and, in turn, how communities impact other entities through their opinions, behaviors, or decision-making processes. Communities have more impact in social networks than do single individuals because a community is a well-organized group, and everyone in the community contributes his or her impact to the whole. Group-organized influence evaluation often identifies users who play significant roles in group work rather than individuals who have great influence. For example, based on a specific topic, by analyzing community influence after community identification, we can find the community that has the greatest effect in spreading the topic and significantly affects further topic diffusion or public opinion supervision. However, the complexity of the inner relationships of a community results in difficulties when evaluating community influence. Because other factors such as the passage of time and dynamic changes in community composition may introduce changes to the community, the influence of the community may also change. Thus, it is essential to accurately represent the influence of a community dynamically.

In this work, we propose a new scheme for evaluating community related influence from a dynamic perspective. We consider that there are two kinds of community related influence: community influence and community-initiated influence that should be evaluated. More specially, we consider that community influence indicates a comprehensive impact formed by all of its members, while the community-initiated influence indicates the impacts from the community to other entities in social network. Therefore, we propose a dynamic community related influence evaluation model (D-CIEM) that includes three types of influence evaluation: dynamic community influence evaluation and both internal and external influence prediction. The main works of this paper are as follows. (1) We propose the D-CIEM framework and its related formal definitions. (2) We propose a dynamic view of community influence evaluation based on information propagation among users - the dynamic feature of D-CIEM. (3) We propose community-initiated dynamic influence evaluation methods, including a method for calculating community influence on internal and external users and influence between communities. We verify the feasibility and effectiveness of the proposed method by experimental results.

We aim to achieve the following contributions.

(1) A new dynamic perspective for influence measurement: the proposed D-CIEM framework is given from a dynamic perspective, while most existing models are given with a static view. That is, most traditional models calculate the influence of entities in social network (including individual user or community) without time dimension. In our paper, we consider the community as a dynamical entity and therefore the influence of community is calculated from three aspects: time passing, membership changes, and community structural instability (details of the three aspects are given in Section 4.3).
(2) Using the topological structure for community influence evaluation: the community is classified according to the structure as overlapping and nonoverlapping communities. Therefore, the influence of community is calculated based on the two different structure features. Therefore, a novelty of our work is that the structural feature is significant for our proposed model.

(3) A multidimension for community influence definition: the influence of community is not just a single value as in traditional works. We consider that the influence of community has three kinds of impacts to different entities: influence to internal user, influence to external user, and influence to community. Hence, we have more perspectives for revealing the influence from a community rather than a simple value.

(4) Evaluating community influence based on more factors: we define many factors that aims to make full use of the features of social network to measure different aspects of the influence.

The remainder of this paper is organized as follows: Section 2 introduces some past related works; Section 3 introduces the D-CIEM framework and related definitions; Section 4 introduces the method for calculating dynamic entity influence; Section 5 introduces the community-initiated influence evaluation methods in the proposed model; Section 6 presents the experiments on datasets and discusses the results of the experiments; and Section 7 summarizes the main work of this paper and provides possible aspects for future research.

\section{Related Work}

Related studies have addressed the topic of influence evaluation or prediction from three aspects: individual influence evaluation, influence maximization, and community influence evaluation. For individual influence evaluation, most works represent social network topology as a graph and then further evaluate influence through users' profiles, behaviors, or relationships. Li [9] proposed user relationship-based information propagation to evaluate an individual's influence on a social network. Cano Basave [13] addressed a user semantic profile-based influence evaluation method using a relation-driven influence ranking. Zhou [14] proposed a top $\mathrm{k}$ influential node mining method by analyzing user's preferences through their labelled documents. N Barbieri [16] proposed a topic-aware user influence propagation model by modeling authoritativeness, influence, and relevance from a topic-aware perspective. Some studies have investigated the area of maximal individual influence to find the top $k$ influential users in certain groups [17-19]. For influence maximization, the important works are aimed at finding a subset of influential users and then evaluating their influence using various algorithms based on an information diffusion model. Lu [20] recursively estimated influence spread using reachable probabilities from node to node. Jendoubi [21] used the theory of belief functions to estimate user influence. Zhu [22] proposed a new ranking metric named SpreadRank generalized by a newly proposed information propagation model called CTMC-ICM. To address influence evaluation in a community, topological information for that community, such 
as the tightness of relationships and structural changes in the community, is significant factors in influence evaluation. Many works have modeled the community and its structure as a subgraph of a static social network [23, 24]. Bozorgi Arastoo et al. [25] proposed a linear threshold model-based method to find influential nodes by exploiting graph community structure. K Lewis [15] found that peer communities in social networks are established based on specific interests or peer influence evaluation. IA Christensen [26] proposed a method to predict group influence by extracting the potential influence from members' interactions in a social network involving a recommender system. In our previous work, we presented methods to predict community influence based on information propagation and link similarity in communities $[27,28]$.

However, the majority of the work from these three aspects has been developed by considering static social networks. Individual influence evaluation often ignores the interactions of individuals and communities. Influence maximization algorithms rely on information diffusion models to evaluate influence. Although community has been considered in some works, their focus was on calculating the influences of individuals in the community rather than the influence of the community as a whole. For community influence evaluation, community topological information may lead to a one-sided analysis influence. Community influence changes due to dynamic changes in user influences in the community, but research has paid little attention to dynamic community influences. Based on the above factors, this paper focuses on methods for evaluating community influence and the community's influence on other entities rather than on evaluating the influence of single individuals. Moreover, influence is considered to be a dynamic instead of a static factor.

\section{Overview of the Proposed Model}

In this section, we first provide an overview of the community related influence evaluation model with dynamic perspective (D-CIEM) and then propose related definitions.

\subsection{Framework of the Dynamic Community Related Influence} Evaluation Model. Similar to other related research, the network topology of a social network can be described based on graph theory. We introduce graph theory to model DCIEM. In D-CIEM, users and their relationships are mapped as vertices and edges. All the entities in a social network can be structured as a graph; a community can be considered as a subgraph of the complete social network graph. The social network and its communities can be described as follows.

(1) Social network graph model: a social network can be represented as a 2 -tuple $G=\langle V, E\rangle$, where $V$ is the set of vertices (users) representing users in the social network and $E$ is the set of edges (relationships) describing the relationships among users.

(2) Community graph model: a community is an organization composed of users who share common values in a social network. A community can be represented as a subgraph of the social network graph model, e.g., $C=\langle C V, C E\rangle$, where $C V \subseteq V$ is the set of users who belong to the community and $C E \subseteq E$ is the set of relationships among the community members.

For convenience in further evaluation, we denote a user as an internal user of a community if that user belongs to the community; otherwise, the user is denoted as an external user to the community. The set of external users of a community is denoted as CEN. Based on the composition of communities, we divide communities into two types: overlapping and nonoverlapping communities. These two types can be described formally as follows:

(a) Overlapping community $(O C)$ : a community is an overlapping community if and only if it contains at least one member who belongs to two or more communities in a social network. An overlapping community can be represented as $O C=\langle M C V, S C V, C E\rangle$, where $M C V \subseteq V$ is the set of members who belong to two or more communities, $S C V \subseteq V$ is the set of members who belong to only one community, and $C E \subseteq E$ is the set of relationships among community members.

(b) Nonoverlapping community $(N C)$ : a community is a nonoverlapping community if and only if all members in the community belong to only one community. A nonoverlapping community can be represented as $N C=\langle S V, C E\rangle$, where $S V \subseteq V$ is the set of members who belong to the community, and $C E \subseteq E$ is the set of relationships among community members.

The OC and NC models present the following properties:

(i) For two users $\left(v_{i}, v_{j}\right)$ in the same community, their relationship is within the community if they have a direct relation, meaning $\left(\forall v_{i}, v_{j} \in C . C V \wedge\right.$ $\left.\exists e\left(v_{i}, v_{j}\right)\right) \longrightarrow\left(e\left(v_{i}, v_{j}\right) \in C . C E\right)$.

(ii) For a relationship $e\left(v_{i}, v_{j}\right)$ in a community, the users of $v_{i}, v_{j}$ belong to the community, meaning $\forall e\left(v_{i}, v_{j}\right) \in$ $C . C E \longrightarrow \forall v_{i}, v_{j} \in C . C V$.

(iii) For an overlapping community, there is no user who belongs to both subsets of $M C V$ and $S C V$, meaning $(O C . M C V \neq \varnothing \wedge$ OC.SCV $\neq \varnothing) \longrightarrow(O C . M C V \cap$ OC.SCV $=\varnothing)$.

(iv) A user in a nonoverlapping community does not belong to another nonoverlapping community, meaning $\left(\exists N C_{i} \neq \varnothing \wedge \exists N C_{j} \neq \varnothing \wedge N C_{i} \neq N C_{j}\right) \longrightarrow$ $\left(N C_{i} \cap N C_{j}=\varnothing\right)$.

(3) D-CIEM: D-CIEM is a model that presents the degree of dynamic influence initiated by a community within the social network.

Details of D-CIEM include both entity influence (a unary value) and community-initiated influence (binary values). Details of the influence degrees are discussed in a later section. The objective function of D-CIEM is $\operatorname{Inf}\left(C_{i}\right)^{t}=$ $f\left(G, C_{i}\right)$, where $\operatorname{Inf}\left(C_{i}\right)^{t}$ denotes the influence degree of community $C_{i}$ at the end of time slice $t$ and function $f\left(G, C_{i}\right)$ denotes the evaluation method based on models of $G$ and $C_{i}$. 
TABLE 1: Nomenclature.

\begin{tabular}{|c|c|c|}
\hline \multicolumn{2}{|c|}{ Symbol } & \multirow{2}{*}{$\begin{array}{l}\text { Description } \\
\text { degree of user influence }\end{array}$} \\
\hline \multirow{2}{*}{ Entity influence } & $\operatorname{Inf}\left(u_{i}\right)^{t}$ & \\
\hline & $\operatorname{Inf}\left(C_{i}\right)^{t}$ & degree of community influence \\
\hline Average intention degree & $\operatorname{aip}()^{t}$ & $\begin{array}{l}\text { the average degree of an entity's inherent intention of propagating } \\
\text { information }\end{array}$ \\
\hline \multirow{3}{*}{ Community-initiated influence } & internal $\operatorname{Inf}\left(C_{i}, u_{j}\right)^{t}$ & the degree of influence of a community on internal users \\
\hline & external_Inf $\left(C_{i}, u_{j}\right)^{t}$ & the degree of influence of a community on external users \\
\hline & $\operatorname{Inf}\left(C_{i}, u_{j}\right)^{t}$ & the degree of influence between communities \\
\hline \multirow{8}{*}{ Factors in the calculations } & $M\left(\operatorname{Info}\left(u_{i}\right) \cdot i n_{j}\right)$ & the information status for a user whether he/she receives it \\
\hline & $\operatorname{pro}\left(\operatorname{Info}\left(u_{i}\right) \cdot i n_{j}\right)$ & the information status for a user whether he/she propagates it \\
\hline & $\operatorname{AcS}\left(u_{i}\right)$ & the number of times a user receives information \\
\hline & $\operatorname{Pro}\left(u_{i}\right)$ & the times a user propagates information \\
\hline & $H_{-} \operatorname{Inf}\left(C_{i}\right)$ & factor of community hybrid internal influence \\
\hline & number $\left(C_{i}\right)$ & factor of community internal user number \\
\hline & $\operatorname{tight}\left(C_{i}\right)$ & factor of community user relationship tightness \\
\hline & $\operatorname{inst}\left(C_{i}\right)^{t_{k}}$ & the attenuation factor for dynamic rule \\
\hline
\end{tabular}
parts.

Our proposed D-CIEM structure includes the following

(1) Part 1: Original Data Preprocessing. In this part, we extract the detailed original data of the social network, including community topological information, user interaction records, and the process of information propagation for further computation in D-CIEM. The process for calculating the influence of nodes is established based on this extracted data, including the intention degree of information propagation, entity influence, and community-initiated influence.

(2) Part 2: Entity Influence Calculation. In D-CIEM, we first calculate the influence of a single user based on that user's average intention degree for information propagation. Two parameters, $A c S$ and Pro, signify the number of times a user receives information and the number of times that user propagates the information. The parameters are calculated based on users' detailed information delivery records. The results of these parameter calculations are combined to calculate the user's average intention degree of information propagation. We evaluate user influence from three aspects, the total number of times a user accepts information, the total number of links connected to users, and the number of interactions a user has with other users external to the community. Community influence is calculated by combining four aspects: the hybrid influence of community internal users, the number of community internal users, the relationship tightness among community internal users, and a dynamic factor for the community.

(3) Part 3: Community-Initiated Influence Calculation. D-CIEM calculates three types of influence degree initiated by the community as defined in Definition 3. The community-initiated influence shows how much influence a community exerts on other entities, including community internal users, community external users, and other communities in the social network.

The detailed structure of D-CIEM is shown in Figure 1.
3.2. Related Definitions. In this section, we present some definitions used in D-CIEM including entity influence and community-initiated influence. We consider two main entities that generate influence in a social network, users and the community, and define their entity influence as follows. The detailed nomenclature in this paper is given in Table 1 .

Definition 1 (entity and entity influence). The user and community in social network are collectively called "Entity" in this paper. And then, entity influence is a unary degree, which reflects the impacts generated by entities, i.e., users and the community in a social network from a dynamic perspective. follows.

The entity influence can be divided into two types as

(1) User Influence $\left(\operatorname{In} f\left(u_{i}\right)^{t}\right)$. User influence is an impact value which indicates the real-time impact degree from a user to other single individuals through his/her social interaction behaviors, e.g., communications, opinions, and emotions in a social network at the end of each time slice.

(2) Community Influence $\left(\operatorname{In} f\left(C_{i}\right)^{t}\right)$. Community influence is an integrated impact value which indicates the realtime impact degree from all members in a community to other entities in the social network at the end of each time slice.

For Definition 1, since community is composed by its individual user members, community related influence is also composed by user influence. That is, user influence is the foundation for community related influence evaluation. In our consideration, we set the value of the influence degree between 0 and 1: the larger the influence value is, the greater the influence of the entity is. Because communities are composed of various users and each user may belong to one or more communities, a user's information propagation behavior plays a definite role in the influence evaluation of 


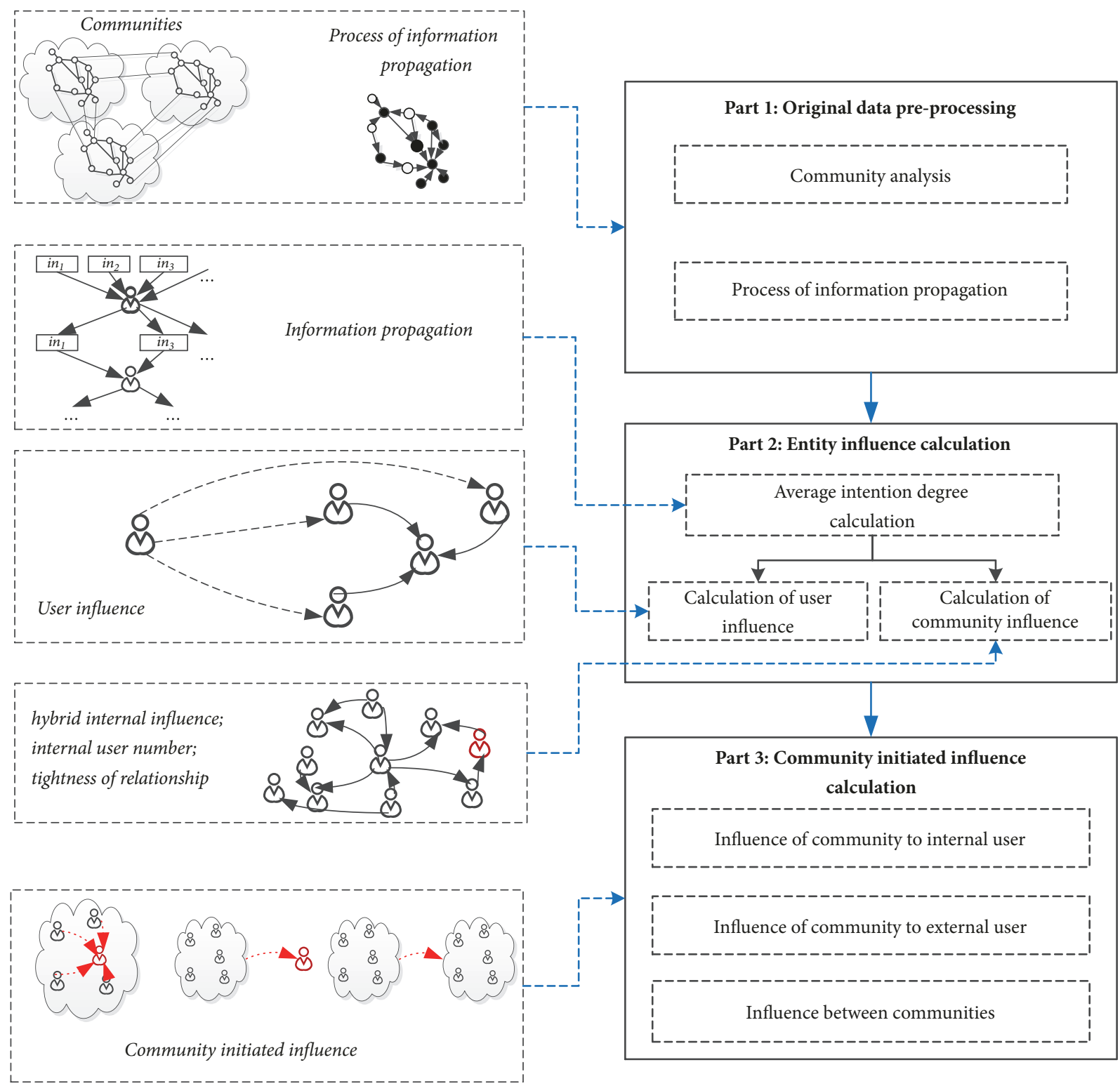

Figure 1: The structure of D-CIEM.

communities. Therefore, we can evaluate influence based on the process of information propagation among users in a social network. To facilitate further evaluation and analysis, we define the average intention degree for propagating information as follows.

Definition 2 (average intention degree for propagating information $\left.\left(\operatorname{aip}()^{t}\right)\right)$. This factor denotes the average degree of an entity's inherent intention of propagating information in a social network at the end of each time slice. This factor aims to describe the probability of propagation information based on entities' inherent willing (the factor of community is a composite number based on the degrees of all the members in the community).
Whether a user decides to propagate information after receiving and seeing it is a significant criterion that can reveal how much influence a user may generate. Therefore, the information propagation behavior of a user, e.g., forwarding, approving, or judging, can contribute to the factor of aip ()$^{t}$. For further calculation, we here signify the information propagated by a user $u_{i}$ as a set $I n f o\left(u_{i}\right)=\left\{i n_{1}, i n_{2}, i n_{3}, \ldots\right\}$, where $i n_{j}$ denotes the $j$-th information propagated by user $u_{i}$. When information $i n_{j}$ is propagated in a social network, there is a subgraph $g\left(i n_{j}\right)$ that describes the propagation paths. We collect the subgraphs formed by the propagation process of Info $\left(u_{i}\right)$ into a set, $g\left(\operatorname{Info}\left(u_{i}\right)\right)=\left\{g\left(i n_{1}\right), g\left(i n_{2}\right), g\left(i n_{3}\right) \ldots\right\}$, where $g\left(i n_{j}\right)$ is the $j$-th subgraph formed by the $j$-th information propagated by user $u_{i}$. 
Moreover, we concern that the community may bring three kinds of influence to different entities: the internal user of a community, the external user of a community, and the other communities. Therefore, we propose another concept, community-initiated influence, to describe the influence degrees that are initiated by a community in a social network.

Definition 3 (community-initiated influence). Communityinitiated influence is a binary degree that describes the influence degrees of a community to other three kinds of entities as follows.

(1) Influence of a Community on Internal Users (internal_ $\left.\operatorname{Inf}\left(C_{i}, u_{j}\right)^{t}\right)$. This factor denotes the influence degree of a community to its internal members who belong to this community at the end of each time slice.

(2) Influence of a Community on External Users (external_Inf $\left.\left(C_{i}, u_{j}\right)^{t}\right)$. This factor denotes the influence degree of a community to its external users who do not belong to this community at the end of each time slice.

(3) Influence between Communities $\left(\operatorname{Inf}\left(C_{i}, C_{j}\right)^{t}\right)$. This factor denotes the influence degree of a community to another different community at the end of time slice.

\section{Entity Influence Calculation Method in D-CIEM}

4.1. Calculation of Average Intention Degree of Information Propagation. This factor comprises all the intention degrees of users propagating information in a community and shows the probability of a community to propagate information. A high value of this factor implies that the community has a high frequency of information propagation, which causes a high relative influence because more interactions between community members and other entities in the social network occur.

To calculate the average intention of information propagation by a community, we first need to evaluate the intention degree of information propagation for a single user. We provide a parameter, $M\left(\operatorname{Info}\left(u_{i}\right) \cdot i n_{j}\right)$, to identify the information status for the user $u_{i}$ after he/she receives information $\operatorname{Info}\left(u_{i}\right) . i n_{j}$ as follows:

$$
\begin{aligned}
& M\left(\text { Info }\left(u_{i}\right) \cdot i n_{j}\right) \\
& = \begin{cases}0 & \text { if user } u_{i} \text { has never received information in } \\
1 & \text { else }\end{cases}
\end{aligned}
$$

To cold start a problem, we set the initial value of a user who has never received information as 0 , and we obtain the propagation statuses of user $u_{i}$ propagating information $\operatorname{Info}\left(u_{i}\right) . i n_{j}$ through graph $g\left(\operatorname{Info}\left(u_{i}\right)\right) . g\left(i n_{j}\right)$. Then, we propose the parameter pro $\left(\operatorname{Info}\left(u_{i}\right) . i n_{j}\right)$, which indicates the status of a user $u_{i}$ who propagates information as follows:

$$
\begin{aligned}
& \operatorname{pro}\left(\operatorname{Info}\left(u_{i}\right) \cdot i n_{j}\right) \\
& = \begin{cases}0 & \text { if user } u_{i} \text { never propagates information Info }\left(u_{i}\right) \cdot i n_{j} \\
1 & \text { else }\end{cases}
\end{aligned}
$$

From the above equation, we can see that if user $u_{i}$ never propagates information $i n_{j}$, the value of $\operatorname{pro}\left(\operatorname{Info}\left(u_{i}\right) \cdot i n_{j}\right)$ remains 0 ; otherwise, user $u_{i}$ has propagated information Info $\left(u_{i}\right) . i n_{j}$. Then, we use two parameters, AcS and Pro, to signify the number of times a user receives information and the times a user propagates information, respectively, which can be calculated as follows:

$$
\begin{aligned}
& \operatorname{AcS}\left(u_{i}\right)=\sum_{u_{k} \in\left(G . V-\left\{u_{i}\right\}\right)} \sum_{i n_{j} \in \operatorname{Info}\left(u_{i}\right)} M\left(\operatorname{Info}\left(u_{i}\right) \cdot i n_{j}\right) \\
& \operatorname{Pro}\left(u_{i}\right)=\sum_{u_{k} \in\left(G . V-\left\{u_{i}\right\}\right)} \sum_{i n_{j} \in \operatorname{Info}\left(u_{i}\right)} \operatorname{pro}\left(\operatorname{Info}\left(u_{i}\right) . i n_{j}\right)
\end{aligned}
$$

In our study, the factor aip $\left(u_{i}\right)^{t}$ is an accumulated value that describes the intention to propagate information by combining all the influences from other users to a user. Therefore, aip $\left(u_{i}\right)^{t}$ of a user $u_{i}$ can be defined as in the following equation:

$$
\begin{aligned}
& \operatorname{aip}\left(u_{i}\right) \\
& =\frac{\theta \times\left(\operatorname{Pro}\left(u_{i}\right) / \operatorname{AcS}\left(u_{i}\right)\right)+(1-\theta) \times \sum_{u_{k} \in\left(G . V-\left\{u_{i}\right\}\right)} \sum_{i n_{j} \in \operatorname{Info}\left(u_{i}\right) \wedge g\left(i n_{j}\right) \in g\left(\operatorname{Info}\left(u_{i}\right)\right)}\left(\sum_{u_{l} \in \operatorname{CV}\left(u_{i}\right)}\left(\operatorname{aip}\left(u_{l}\right) \times w\left(u_{l}\right)\right)+\sum_{u_{r} \in \operatorname{CEN}\left(u_{i}\right)}\left(\operatorname{aip}\left(u_{r}\right) \times w\left(u_{r}\right)\right)\right)}{n u m\left(g\left(\operatorname{Info}\left(u_{i}\right)\right)\right)} \\
& +\frac{\left|\operatorname{Info}\left(u_{i}\right)\right|}{\operatorname{num}\left(g\left(\operatorname{Info}\left(u_{i}\right)\right)\right)} \\
& w\left(u_{l}\right)=\frac{1}{\text { outdegree }(e)}, \\
& w\left(u_{r}\right)=\frac{t_{\text {out } \rightarrow \text { in }}}{t_{\text {in } \rightarrow \text { out }} \times \text { num }_{\text {C-out }}} \times \frac{1}{\text { outdegree }\left(u_{r}\right)}
\end{aligned}
$$

where $\operatorname{aip}\left(u_{i}\right)$ is the value of factor for user $u_{i}, \theta$ is a weight between 0 and $1, \operatorname{Pro}\left(u_{i}\right)$ is the total number of times that user $u_{i}$ propagated information, $\operatorname{AcS}\left(u_{i}\right)$ is the total number of times that user $u_{i}$ received information, and $C V\left(u_{i}\right)$ and $C E N\left(u_{i}\right)$ are the set of internal and external users, respectively, who have direct links to user $u_{i}$. Here, $w\left(u_{l}\right)$ and 


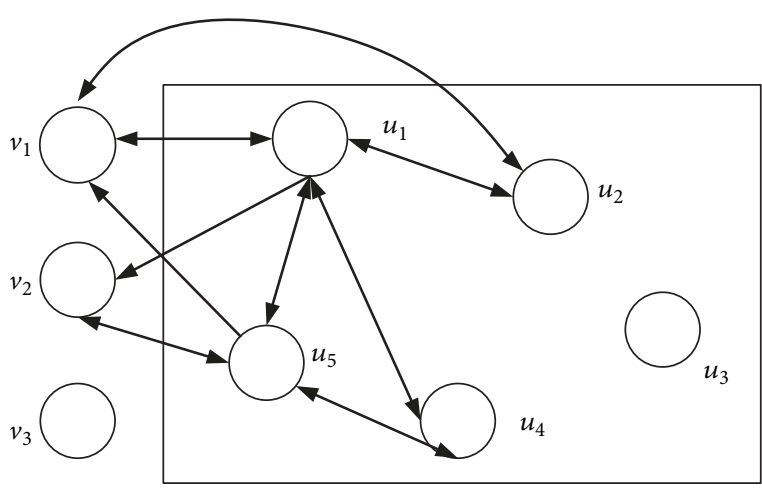

(a) The initial relationships of users

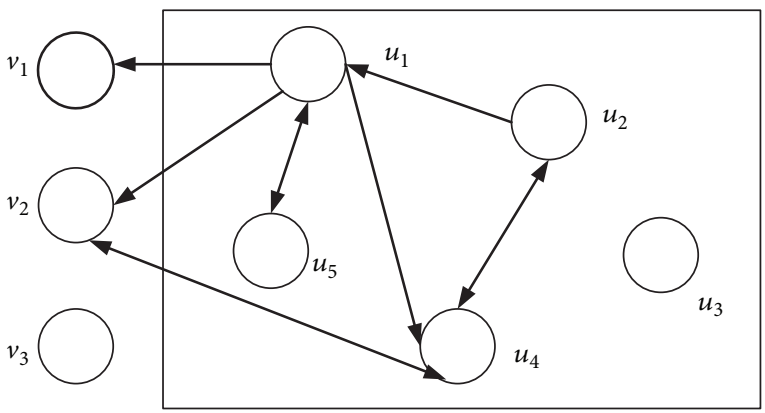

(c) Information propagation graph published be $u_{1}$

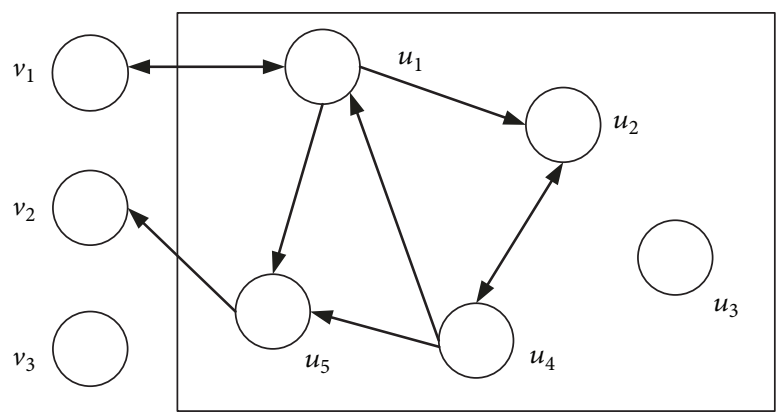

(b) Information propagation graph published be $u_{1}$

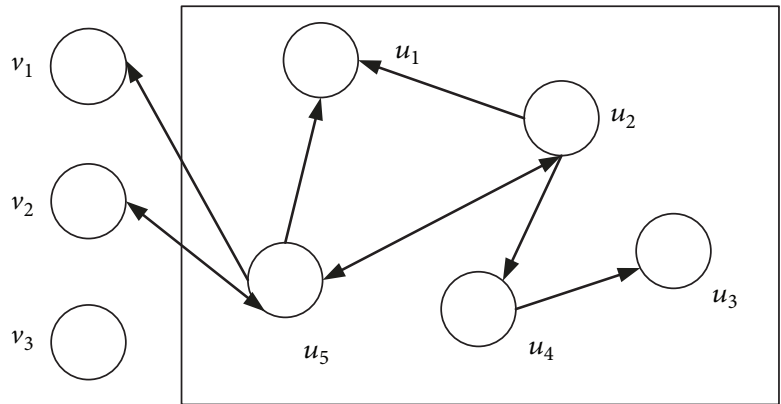

(d) Information propagation graph published be $u_{2}$

FIgURE 2: An example of AIP factor calculation.

$w\left(u_{r}\right)$ represent the weights of user $u_{l} \in C V\left(u_{i}\right)$ and user $u_{r} \epsilon$ $C E N\left(u_{i}\right)$, respectively, and they are measured by the numbers of users' out-degrees, and num $\left(g\left(\operatorname{Info}\left(u_{i}\right)\right)\right)$ denotes the total number of information propagation graphs of $g\left(\operatorname{Info}\left(u_{i}\right)\right)$. There is an influence coefficient for the ratio of external users to internal users in the calculation of $w\left(u_{r}\right): t_{\text {out } \rightarrow \text { in }}$ denotes the total number of external users connected to internal users in all the propagation graphs, $t_{i n \rightarrow o u t}$ denotes the total number of internal users connected to external users in all the propagation graphs; and num $\mathrm{C}_{\text {-out }}$ denotes the number of external users. We assume that the initial default value of aip $\left(u_{i}\right)$ is 1 for cold-start problems. To justify the factor, we here give an example of AIP factor calculation as shown in Figure 2.

Figure 2 shows the calculation example of aip $\left(u_{i}\right)$ factor. In Figure 2, there are five community internal users $\left(u_{1}, u_{2}, u_{3}, u_{4}\right.$, and $\left.u_{5}\right)$ and three community external users $\left(v_{1}, v_{2}\right.$, and $\left.v_{3}\right)$, all connected by their relationships. Assume that the initial values of a user's aip $\left(u_{i}\right)$ are 1 in Figure 2(a). As shown in Figures 2(b)-2(d), we obtain $t_{\text {out } \rightarrow \text { in }} /\left(t_{\text {in } \rightarrow \text { out }} \times\right.$ num $\left._{C-\text { out }}\right)=3 /(7 \times 3)=1 / 7, \theta=0.6$. Then, we have the following calculations for the $\operatorname{aip}\left(u_{i}\right)$ factor of all users in Figure 3.

(1) aip $\left(u_{1}\right)$ : in Figures 2(b)-2(d), we have $\operatorname{num}\left(g\left(\operatorname{Info}\left(u_{i}\right)\right)\right)=3$. For user $u_{1}$, there are two information propagation graphs, which are visible in Figures $2(b)-2(d)$. Thus, we have set Info $\left(u_{1}\right)$, and $\left|\operatorname{Info}\left(u_{1}\right)\right|=2$. In Figure 2(c), $u_{1}$ has received information; thus, we obtain $\operatorname{AcS}\left(u_{1}\right)=1$. Then, we obtain $\operatorname{Pro}\left(u_{1}\right)=0$. In Figure 2(d), because the out-degree value of $u_{1}$ is 0 , we can calculate aip $\left(u_{1}\right)$ in Figure 2(d) as follows:

$$
\begin{aligned}
& C V\left(u_{1}\right)=\left\{u_{2}, u_{5}\right\}, \\
& \operatorname{CEN~}\left(u_{1}\right)=\varnothing ; \\
& \begin{array}{l}
w\left(u_{2}\right)=\frac{1}{2}, \\
w\left(u_{5}\right)=\frac{1}{4} . \\
\text { aip }\left(u_{1}\right) \\
\quad=\frac{0.6 \times(0 / 1)+0.4 \times(1 \times(1 / 2)+1 \times(1 / 4))}{3}+\frac{2}{3} \\
\quad=\frac{23}{30}
\end{array}
\end{aligned}
$$

(2) aip $\left(u_{2}\right)$ : Similar to the calculation of aip $\left(u_{2}\right)$, we have a set of $\operatorname{Info}\left(u_{2}\right)$ and $\left|\operatorname{Info}\left(u_{2}\right)\right|=1$. From Figures 2(b)-2(c), the values of the factors $\operatorname{AcS}\left(u_{2}\right)$ and $\operatorname{Pro}\left(u_{2}\right)$ are calculated as 2 and 1 , respectively. Thus, we obtain $\operatorname{aip}\left(u_{2}\right)$ as follows:

$$
\begin{aligned}
C V\left(u_{2}\right) & =\left\{u_{2}, u_{4}\right\}, \\
C E N\left(u_{2}\right) & =\varnothing ; \\
w\left(u_{2}\right) & =1, \\
w\left(u_{4}\right) & =\frac{1}{3} .
\end{aligned}
$$




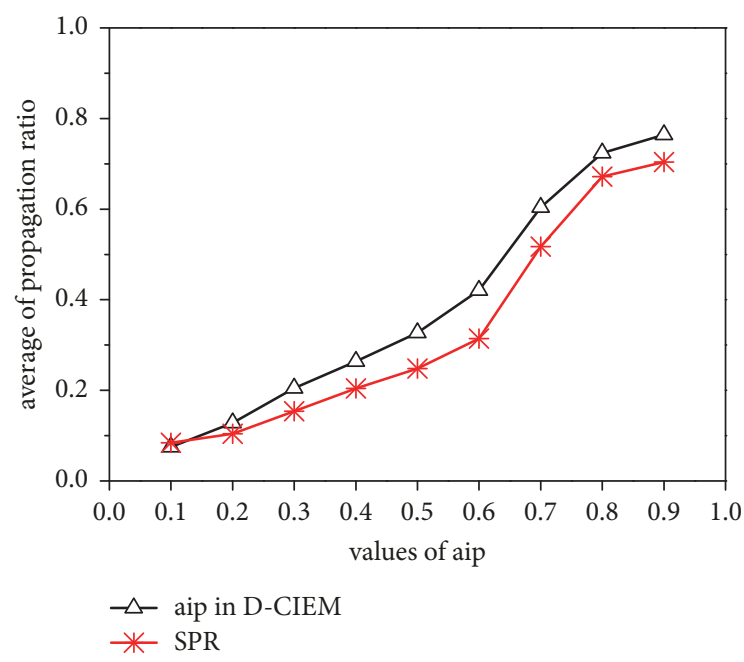

(a)

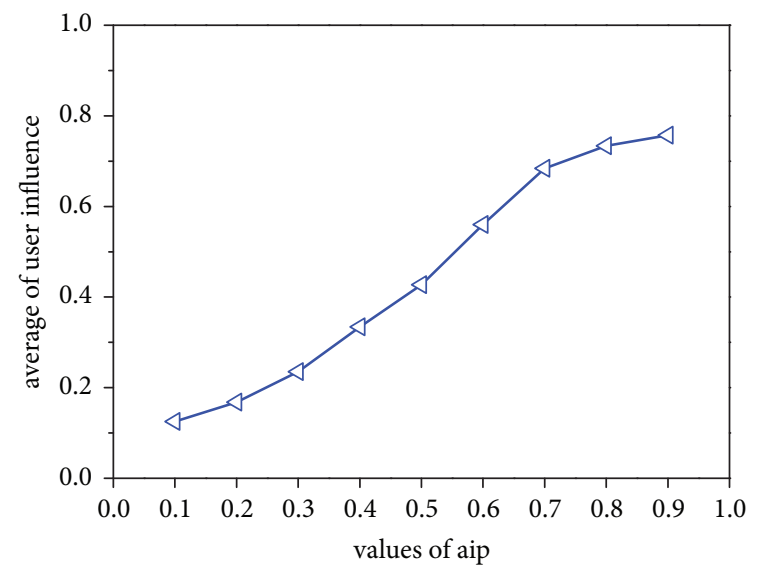

(b)

FiguRE 3: Performance analysis of the proposed average intention of propagating information.

$$
\begin{aligned}
\operatorname{aip}\left(u_{2}\right)= & \frac{0.6 \times(1 / 2)+0.4 \times(1 \times 1+1 \times(1 / 3))}{3} \\
& +\frac{1}{3}=\frac{1}{18} .
\end{aligned}
$$

We then calculate the $\operatorname{aip}\left(u_{i}\right)$ factors for users $u_{3}, u_{4}$, and $u_{5}$ as follows:

$$
\begin{aligned}
& \operatorname{aip}\left(u_{3}\right):\left\{\begin{array}{l}
\operatorname{Pro}\left(u_{3}\right)=0+0+0=0 \\
\operatorname{AcS}\left(u_{3}\right)=0+0+1=1 \\
\operatorname{aip}\left(u_{3}\right)=\frac{0.6 \times 0+0.4 \times 0}{3}+0=0
\end{array}\right. \\
& \operatorname{aip}\left(u_{4}\right):\left\{\begin{array}{l}
\operatorname{Pro}\left(u_{4}\right)=1+1+1=3 ; \operatorname{AcS}\left(u_{4}\right)=1+1+1=3 \\
\operatorname{CV}\left(u_{4}\right)=\left\{u_{2}\right\}, \operatorname{CEN}\left(u_{4}\right)=\varnothing, w\left(u_{2}\right)=1 \\
\operatorname{CV}\left(u_{4}\right)=\left\{u_{1}, u_{2}\right\}, \operatorname{CEN}\left(u_{4}\right)=\left\{v_{2}\right\} ; w\left(u_{1}\right)=\frac{1}{3}, w\left(u_{2}\right)=\frac{1}{2}, w\left(v_{2}\right)=\frac{1}{7} \times 1=\frac{1}{7} \\
\operatorname{CV}\left(u_{4}\right)=\left\{u_{2}\right\}, \operatorname{CEN}\left(u_{4}\right)=\varnothing, w\left(u_{2}\right)=\frac{1}{3} \\
\operatorname{aip}\left(u_{4}\right)=\frac{(0.6 \times(3 / 3)+0.4 \times(1 \times 1+(1 \times(1 / 3)+1 \times(1 / 2)+1 \times(1 / 7)))+1 \times(1 / 3))}{3}+0=\frac{32}{63}
\end{array}\right. \\
& \operatorname{aip}\left(u_{5}\right):\left\{\begin{array}{l}
\operatorname{Pro}\left(u_{5}\right)=0+1+1=2 ; \operatorname{AcS}\left(u_{5}\right)=1+1+1=3 \\
\operatorname{CV}\left(u_{5}\right)=\left\{u_{1}\right\}, \operatorname{CEN}\left(u_{5}\right)=\varnothing, w\left(u_{2}\right)=\frac{1}{3}, w\left(u_{4}\right)=\frac{1}{3} \\
\operatorname{CV}\left(u_{5}\right)=\left\{u_{1}\right\}, \operatorname{CEN}\left(u_{5}\right)=\varnothing, w\left(u_{1}\right)=\frac{1}{3} \\
\operatorname{CV}\left(u_{5}\right)=\left\{u_{2}\right\}, \operatorname{CEN}\left(u_{5}\right)=\left\{v_{2}\right\} ; w\left(u_{2}\right)=\frac{1}{3}, w\left(v_{2}\right)=\frac{1}{7} \times 1=\frac{1}{7} \\
\operatorname{aip}\left(u_{5}\right)=\frac{(0.6 \times(2 / 3)+0.4 \times((1 \times(1 / 3)+1 \times(1 / 3))+1 \times(1 / 3)+(1 \times(1 / 3)+1 \times(1 / 7))))}{3}+0=\frac{38}{105}
\end{array}\right.
\end{aligned}
$$


4.2. User Influence Evaluation. According to the strength of a user's identification with a community, he or she may be influenced by others-both internal and external users-to different degrees. In this study, we use a weighted average method to evaluate user influence. We use the weight parameters to distinguish the different influences of users. The weights are determined by the following: the total number of times a user accepts information, the total number of links connected to a user, and the number of a user's interactions with community external users. User influence can be calculated as follows:

$$
\begin{aligned}
& \operatorname{Inf}\left(u_{i}\right)=\xi \sum_{\operatorname{in}_{j} \in \operatorname{Info}\left(u_{i}\right)}\left(\operatorname{sum}_{{\text {in } i n_{j}}}+\beta \times \text { sum }_{\text {out } i n_{j}}\right) \\
& \xi=\frac{1}{n u m_{S N}|S(u)|} \\
& \text { sum }_{\text {inin }_{j}}=\sum_{v_{j} \in\left\{C V\left(u_{j}\right)-u\right\}} M\left(\operatorname{Info}\left(v_{j}\right) \cdot i n_{j}\right) \\
& \beta=\frac{t_{\text {in } \longrightarrow \text { out }}}{t_{\text {ac.out }} \times \text { num }_{\text {C-out }}} \\
& \operatorname{sum}_{\text {out } \text { in }_{j}}=\sum_{v_{k} \in \operatorname{CEN}\left(v_{k}\right)} M\left(\operatorname{Info}\left(v_{k}\right) \cdot \text { in }_{j}\right) \text {, }
\end{aligned}
$$

where $\operatorname{Inf}\left(u_{i}\right)$ is the $U I$ of user $u_{i}$; sum in in $_{j}$ is the number of internal users who are influenced by the information Info $\left(u_{i}\right) . i n_{j}$, and sum $_{\text {out } \_n_{j}}$ is the number of external users who are influenced by information $\operatorname{Info}\left(u_{i}\right) \cdot i n_{j}$. Here, $\beta$ is an influence coefficient that measure the impacts of user $u_{i}$ on his or her community external users; $t_{\text {in } \rightarrow o u t}$ denotes the number of internal users in the community connected to external users, $t_{\text {ac.out }}$ denotes the total number of times external users accepted the information, num $_{\mathrm{C}-\text { out }}$ denotes the number of community external users, $\xi$ is the influence factor, $|G . V|$ denotes the total number of users in the social network, and $\left|\operatorname{Info}\left(u_{i}\right)\right|$ denotes the amount of information published by a user $u_{i}$. If the user has never published information, we set $\operatorname{In} f\left(u_{i}\right)$ to 0.1 .

An example follows.

In Figures $2(\mathrm{~b})-2(\mathrm{~d})$, we know that $\beta=7 /(7 \times 3)=1 / 3$.

$\operatorname{Inf}\left(u_{1}\right)$ : we have the following parameters: $|G . V|=$ 8, $\left|\operatorname{Info}\left(u_{1}\right)\right|=2, \xi=1 / 8^{2}$. Consequently, we can calculate $\operatorname{Inf}\left(u_{1}\right)$ in Figure 3 as follows:

$$
\begin{array}{rl}
\text { sum }_{{\text {in } i n_{j}}_{j}} & 3, \\
\text { sum }_{\text {out } \_n_{j}} & =2, \\
\operatorname{Inf}\left(u_{1}\right) & =\frac{1}{8^{2}} \times\left(\left(3+\frac{1}{3} \times 2\right)+\left(3+\frac{1}{3} \times 2\right)\right) \\
& =\frac{11}{96} .
\end{array}
$$

$\operatorname{Inf}\left(u_{2}\right)$ : we have the following parameters: $|G . V|=$ $8,\left|\operatorname{Info}\left(u_{2}\right)\right|=1, \xi=1 / 8^{1}$. Therefore, we can calculate $\operatorname{Inf}\left(u_{2}\right)$ in Figure 3 as follows:

$$
\begin{aligned}
& \text { sum }_{\text {in_in }_{j}}=5, \\
& \text { sum }_{\text {out_in }_{j}}=2 . \\
& \operatorname{Inf}\left(u_{2}\right)=\frac{1}{8}\left(5+\frac{1}{3} \times 2\right)=\frac{17}{24} .
\end{aligned}
$$

(3) Because users $u_{3}, u_{4}, u_{5}$ have never published information, they each have default values of $\operatorname{Inf}\left(u_{i}\right)$ at 0.1 .

As mentioned above, the influence degree of a user in DCIEM is considered to be dynamic, changing as time passes, rather than a static value. We consider the evolutionary process over time in the user influence degree calculation by assuming that older influences attenuate more as time passes. Because the community influence degree in D-CIEM is an aggregated degree based on its members' influence degrees, community influence also maintains a dynamic property. Therefore, we propose a dynamic prediction method based on an aging algorithm to describe the dynamic feature of influence. We provide an attenuation coefficient $\omega \in[0,1]$ to measure the attenuation in influence degree as time passes. Suppose the influence degree of user $u_{i}$ in the $k$-th time slice $t_{k}$ is $\operatorname{Inf}\left(u_{i}\right)^{t_{k}}$; then, that user's influence degree at the end of time slice $t_{n}$ is calculated as follows:

$$
\operatorname{Inf}\left(u_{i}\right)^{t_{n}}=\frac{1}{n} \sum_{k=0}^{n}\left(\operatorname{Inf}\left(u_{i}\right)^{t_{k}} \times \omega^{n-k}\right) .
$$

In the above equation, the influence degree of the user in the $k$-th time slice, $t_{k}$, would decrease as time passes.

4.3. Community Influence Evaluation. Community influence is considered to be an integrated value affected by both a community's internal users and external users. We consider four factors when measuring dynamic community influence: the hybrid influence of community internal users, the number of community internal users, the relationship tightness among community internal users, and a dynamic community factor. The community influence calculation based on the above four factors is detailed as follows.

4.3.1. Factor of Hybrid Internal Influence. The hybrid internal influence of community internal users $H \operatorname{Inf}\left(C_{i}\right)$ is calculated based on the values of $\operatorname{aip}\left(u_{i}\right)$ and $\operatorname{Inf}\left(u_{i}\right)$ and defined as follows:

$$
H \_\operatorname{Inf}\left(C_{i}\right)=\frac{\sum_{u_{j} \in C_{i} \cdot C V}\left(\operatorname{aip}\left(u_{j}\right) \times \operatorname{Inf}\left(u_{j}\right)\right)}{\left|C_{i} \cdot C V\right|},
$$

where $C_{i} \cdot C V$ is the set of internal users in community $C_{i}$.

4.3.2. Factor of Internal User Number. The number of internal users in the community plays a vital role in calculating community influence. Generally, a larger number of community internal users implies a higher community influence. Based 
on this idea, we use the following calculation for the internal user number factor:

$$
\text { number }\left(C_{i}\right)=\frac{\left|C_{i} \cdot C V\right|}{\max (C)},
$$

where $\left|C_{i} \cdot C V\right|$ denotes the number of internal users in the community $C_{i}$ and the function $\max (C)$ is the maximum number of communities in the social network.

4.3.3. Factor of User Relationship Tightness. User relationship tightness reflects the closeness degree of community internal users. Closer relationships among community internal users may result in a higher community influence. We describe the factor of user relationship tightness, noted as $\operatorname{tight}\left(C_{i}\right)$, by the users' in-degree values:

$$
\operatorname{tight}\left(C_{i}\right)=\frac{\sum_{u_{j} \in C_{i} . C V} \operatorname{in} \operatorname{link}\left(u_{j}\right)}{\left|C_{i} \cdot C V\right| \times\left(\left|C_{i} \cdot C V\right|-1\right)},
$$

where in $\operatorname{link}\left(u_{j}\right)$ denotes the numbers of relationships from community internal user $u_{j}$ to other internal users in the community and $\left|C_{i} . C V\right|$ denotes the number of internal users in community $C_{i}$. From the example in Figure 3(a), we can obtain $\operatorname{tight}\left(C_{i}\right)$ as follows:

$$
\operatorname{tight}\left(C_{i}\right)=\frac{14}{20}=0.7
$$

4.3.4. Dynamic Community Factor. In D-CIEM, a community is also a dynamic entity because the community composition changes dynamically over time. Three aspects can dynamically change community influence: time passing, membership changes, and community structural instability. To measure community influence while considering these dynamic features, we set the following rules for D-CIEM:

(i) Time passing rule: the effect of time passing on community influence is shown by the dynamic characteristics of user influence. The hybrid internal influence of a community is calculated based on dynamic user influence; hybrid internal influence can therefore be seen as a dynamic value. Community influence should therefore be updated as user influence changes to satisfy the time passing rule.

(ii) Membership change rule: the membership of a community changes as users join or leave the community. Membership changes certainly alter the community influence. Therefore, the membership change rule for D-CIEM ensures that when a member joins or leaves the community, the D-CIEM recalculates the community influence. Moreover, when a member changes his or her internal relationships with other members in the community, the D-CIEM also recalculates the community influence. To reduce the computational load, D-CIEM only processes the membership change rule at the end of each time slice.

(iii) Structural instability rule: in D-CIEM, an unstable community structure also implies an unstable influence. In D-CIEM, the attenuation factor $\operatorname{inst}\left(C_{i}\right)^{t_{k}}$ in the $k$-th time slice $t_{k}$ for this rule is as follows:

$$
\begin{gathered}
\operatorname{inst}\left(\mathrm{C}_{i}\right)^{t_{k}}=\frac{1}{2}\left[\sqrt{\frac{\sum_{k=0}^{n}\left(\left|C_{i} \cdot C V\right|^{t_{k}}-\left|\overline{C_{i} \cdot C V}\right|\right)^{2}}{n}}\right. \\
\left.+\sqrt{\frac{\sum_{k=0}^{n}\left(\sum_{u_{j} \in C_{i} \cdot C V} \operatorname{in} \operatorname{link}\left(u_{j}\right)^{t_{k}}-\overline{\operatorname{inlink}\left(C_{i}\right)}\right)^{2}}{n}}\right]
\end{gathered}
$$

where $\left|C_{i} \cdot C V\right|^{t_{k}}$ denotes the number of members in the community $C_{i}$ at time slice $t_{k},\left|\overline{C_{i} \cdot C V}\right|$ is the average number of community members in all past time quanta, $\sum_{u_{j} \in C_{i} . C V}$ in $\operatorname{link}\left(u_{j}\right)^{t_{k}}$ denotes the total number of internal relationships among members in the community at time slice $t_{k}$, and $\overline{i n \_l i n k\left(C_{i}\right)}$ is the average of the relationships in community $C_{i}$ over all past time quanta.

The community influence calculation method at the end of the $k$-th time slice $t_{k}$ according to the above rules is as follows:

$$
\begin{aligned}
\operatorname{Inf}\left(\mathrm{C}_{i}\right)^{t_{k}} & \\
= & \frac{1}{3}\left[H \operatorname{Inf}\left(\mathrm{C}_{i}\right)^{t_{k}}+\text { number }\left(C_{i}\right)^{t_{k}}+\text { tight }\left(\mathrm{C}_{i}\right)^{t_{k}}\right] \\
& \times \tau^{\text {inst }\left(\mathrm{C}_{i}\right)^{t_{k}}}
\end{aligned}
$$

where $\tau$ is a parameter for the attenuation factor that reflects a value between 0 and 1 . A lower value of inst $\left(C_{i}\right)^{t_{k}}$ results in less attenuation of community influence in D-CIEM. Similar to the calculation for user influence, the dynamic community influence calculation after $n$ time quanta is as follows:

$$
\operatorname{Inf}\left(C_{i}\right)^{t_{n}}=\frac{1}{n} \sum_{k=0}^{n}\left(\operatorname{Inf}\left(C_{i}\right)^{t_{k}} \times \omega^{n-k}\right) \text {. }
$$

\section{Community-Initiated Influence Calculation in D-CIEM}

5.1. Community Influence on Internal Users. Community influence over internal users reflects the impact of a community on its internal users, denoted as internal_Inf $\left(C_{i}, u_{j}\right)^{t}$ and represents a comprehensive influence degree for users in the community. Community internal users are impacted by all the information propagated by other internal users of the community. Thus, there is a community internal user $u_{j} \epsilon$ $C_{i} \cdot C V$, where $C_{i} \cdot C V$ is the set of internal users in community $C_{i}$. Assume that the other internal users $u_{k} \in C_{i} . C V \wedge u_{k} \neq$ $u_{j}$ have the information propagation set $\operatorname{Info}\left(u_{j}\right)$. Then, the internal_Inf $\left(C_{i}, u_{j}\right)^{t}$ of this community to its internal user $u_{j}$ at the end of time slice $t$ is calculated as follows: 


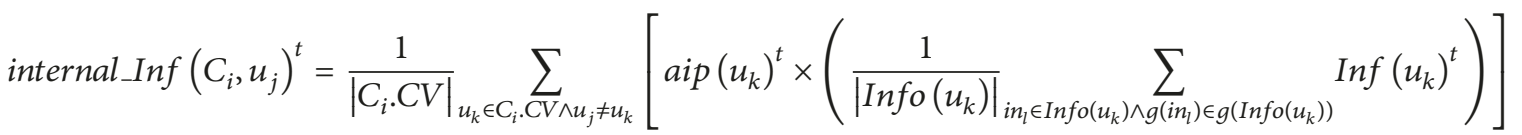

$$
\begin{aligned}
& \inf \left(u_{k}\right)^{t}= \begin{cases}1 & u_{j} \text { is influenced by information propagation process } g\left(i n_{l}\right) \text { in time quantum } t \\
0 & \text { else }\end{cases}
\end{aligned}
$$

where the function $\inf \left(u_{k}\right)$ is used to signify whether $u_{j}$ is influenced by other internal users. After $n$ time quanta, the community's influence on an internal user can be calculated as follows:

$$
\begin{aligned}
& \text { internal_Inf }\left(C_{i}, u_{j}\right)^{t_{n}} \\
& \quad=\frac{1}{n} \sum_{k=0}^{n}\left(\text { internal_Inf }\left(C_{i}, u_{j}\right)^{t_{k}} \times \omega^{n-k}\right) .
\end{aligned}
$$

5.2. Community Influence on External Users. This factor reflects the influence of a community on its external users, which has two aspects: the internal influence of the external user's linked community and community influence. There is an external user $v$ and a community $C_{i}$ whose internal user set is $C_{i} . C V$. Assume that the linked community internal user set of external user $v$ is $\operatorname{Linked}(v) \in C_{i} . C V$, and the community influence of $C_{i}$ is $\operatorname{Inf}\left(\mathrm{C}_{i}\right)^{t_{n}}$. Then, the value of influence from $C_{i}$ to $v$ at the end of time slice $t$, noted as external $\operatorname{Inf}\left(C_{i}, u_{j}\right)^{t_{n}}$, is defined as follows:

$$
\begin{gathered}
\text { external_Inf }\left(C_{i}, v\right)^{t}=\omega \times \operatorname{Inf}\left(\mathrm{C}_{i}\right)^{t}+(1-\omega) \\
\cdot\left[\frac { | \operatorname { L i n k e d } ( v ) | } { | C _ { i } \cdot C V | } \sum _ { u _ { j } \in \operatorname { L i n k e d } ( v ) } \left(\operatorname{aip}\left(u_{j}\right)^{t}\right.\right. \\
\left.\left.\times\left(\sum_{i n_{l} \in \operatorname{Info}\left(u_{j}\right) \wedge g\left(i n_{l}\right) \in g\left(\operatorname{Info}\left(u_{j}\right)\right)} \inf (v)\right)^{t}\right)\right]
\end{gathered}
$$

where $\inf (v)$ is used to signify whether external user $v$ is influenced by information propagated by community internal users, the calculation method is the same as in (19), and $\omega \in[0,1]$ is a weight given in advance. Based on an empirical analysis, the value of $\omega$ is set to 0.4 in our later experiments. The community influence on external user after $n$ time quanta can be calculated as follows:

$$
\begin{aligned}
& \text { external_Inf }\left(C_{i}, u_{j}\right)^{t_{n}} \\
& =\frac{1}{n} \sum_{k=0}^{n}\left(\text { external_Inf }\left(C_{i}, u_{j}\right)^{t_{k}} \times \omega^{n-k}\right) .
\end{aligned}
$$

5.3. Influence between Communities. We discuss the influence between communities for both nonoverlapping and overlapping communities.
5.3.1. Influence between Nonoverlapping Communities. Assume that two communities (noted as $N C_{A}$ and $N C_{B}$ ) are both nonoverlapping communities. In this case, the influence degree from $N C_{A}$ to $N C_{B}$ is determined by two factors, the community influence of $N C_{A}$ and the number of internal users in $N C_{B}$ influenced by community $N C_{A}$. Then, the influence degree from $N C_{A}$ to $N C_{B}$ in the $k$-th time slice $t_{k}$, denoted as $\operatorname{Inf}\left(N C_{A}, N C_{B}\right)^{t_{k}}$, is as follows:

$$
\begin{aligned}
\operatorname{Inf}\left(N C_{A}, N C_{B}\right)^{t_{k}} \\
=\omega \operatorname{Inf}\left(N C_{A}\right)^{t_{k}} \\
\quad+(1-\omega) \frac{\sum_{v_{l} \in N C_{B} \cdot C V} \text { external Inf }\left(N C_{A}, v_{l}\right)^{t_{k}}}{\left|N C_{B} \cdot C V\right|}
\end{aligned}
$$

where external_Inf $\left(N C_{A}, v_{l}\right)^{t_{k}}$ is the community influence from $N C_{A}$ to its external users who belong to community $N C_{B}$.

5.3.2. Influence between Overlapping Communities. For overlapping communities, we calculate the influence by separating the community into two subcommunities, which include user sets of $M C V \subseteq O C . C V$ and $S C V \subseteq$ OC.CV. For the subcommunity composed of user set $M C V \subseteq O C . C V$, we obtain the community internal user influence for each user, while, for the subcommunity composed by user set $S C V \subseteq O C . V$, we obtain the influence of two nonoverlapping communities. Assume that there are two communities $\left(O C_{A}\right.$ and $O C_{B}$ ) and that they are overlapping communities. Thus, there are two subcommunities $S C V\left(O C_{B}\right)=O C_{B} \cdot C V-$ $O C_{A} \cdot C V$ and $M C V\left(O C_{B}\right)=O C_{A} \cdot C V \cap O C_{B} \cdot C V$. In this case, the influence degree from $O C_{A}$ to $O C_{B}$ is determined by the community influence of $O C_{A}$, the influence from $O C_{A}$ to $S C V\left(O C_{B}\right)$, and the influence degree from $O C_{A}$ to users in $M C V\left(O C_{B}\right)$. The community-initiated influence for overlapping communities is defined as follows:

$$
\begin{gathered}
\operatorname{Inf}\left(O C_{A}, O C_{B}\right)^{t_{k}}=\frac{1}{2} \times\left[\operatorname{Inf}\left(O C_{A}\right)^{t_{k}}+\frac{1}{2}\right. \\
\times\left(\operatorname{Inf}\left(O C_{A}, \operatorname{SCV}\left(O C_{B}\right)\right)^{t_{k}}\right. \\
\left.\left.+\frac{\sum_{v_{l} \in M C V\left(O_{B}\right)} \text { internal_Inf }\left(O C_{A}, v_{l}\right)^{t_{k}}}{\left|M C V\left(O C_{B}\right)\right|}\right)\right]
\end{gathered}
$$


TABLE 2: Detail of our experiment.

\begin{tabular}{lccc}
\hline Contents of network & Value & Community statistics & Value \\
\hline Nodes & 6,856 & Number of communities & 461 \\
Edges & 142,544 & Average number of members of communities & 26.7 \\
Maximal degree & 116 & Average number of internal edges of communities & 407 \\
Average degree & 21 & Average number of external edges of communities & 183 \\
Diameter & 11 & Related positive behaviors in social network & Posting, approving, labeling, forwarding, commenting \\
& & The length of a time slice & 7 days \\
\hline
\end{tabular}

where $\operatorname{Inf}\left(O C_{A}, S C V\left(O C_{B}\right)\right)^{t_{k}}$ is the influence degree from community $O C_{A}$ to subcommunity $\operatorname{SCV}\left(C_{B}\right)$ at the end of the $k$-th time slice $t_{k}$, and internal_Inf $\left(O C_{A}, v_{l}\right)^{t_{k}}$ is the influence degree from community $O C_{A}$ to its internal user $v_{l} \in \operatorname{MCV}\left(C_{B}\right)$ at the end of the $k$-th time slice $t_{n}$.

The above two kinds of influence between communities after $n$ time quanta can be calculated as follows:

$$
\begin{aligned}
& \operatorname{Inf}\left(N C_{A}, N C_{B}\right)^{t_{n}} \\
& =\frac{1}{n} \sum_{k=0}^{n}\left(\operatorname{Inf}\left(N C_{A}, N C_{B}\right)^{t_{k}} \times \omega^{n-k}\right) \\
& \operatorname{Inf}\left(O C_{A}, O C_{B}\right)^{t_{n}} \\
& =\frac{1}{n} \sum_{k=0}^{n}\left(\operatorname{Inf}\left(O C_{A}, O C_{B}\right)^{t_{k}} \times \omega^{n-k}\right) .
\end{aligned}
$$

5.4. Complexity Analysis. For entity influence, the computation complex is as follows: (1) given a community and its user set $C=\langle C V, C E\rangle$, let $n=|C \cdot C V|, m=|C . C E|$, and there is $k$ information that had been propagated in past. The user influence is calculated based on the information propagation status and its related users. Therefore, the computation complexity of user influence is $O(\mathrm{~km})$; (2) the community influence is calculated based on all members in it, then its computation complexity is $\mathrm{O}(\mathrm{knm})$.

For community influence on internal users, the degree is calculated based on all the members who can impact the internal user and the information propagated in community; therefore, its computation complexity is $O(\mathrm{knm})$. Likewise, the computation complexity of community influence on external users is also $\mathrm{O}(\mathrm{knm})$. For influence between communities, there are two communities included in influence computation. Our proposed model first calculates the community influence respectively and then obtains the influence between communities. Therefore, the complexity is mainly determined by the first step and is also $O(\mathrm{knm})$.

\section{Experiment and Results Analysis}

In this section, we present the experiments used to verify our proposed approach. The simulation environment is as follows:

(1) Simulation Platform. To test the performance of our proposed method, we developed a prototype that can simulate users and their interaction behaviors based on prototype configurations. In the prototype, users record their interactions with others according to a set of behaviors, such as posting information, sending comments, labelling posts, forwarding posts, and approving posts.

(2) Datasets. In our scenario, the data was sourced from Sina Weibo because it included the necessary records (e.g., forwarding, labelling, approving, and following) for the trust and influence calculations in this study. We collected information manually from the sina.com micro-blog for a span of three months through the API interface provided by sina.com. Our data included 6,856 IDs and more than 400,000 information propagation-related records. From these datasets, we manually collected 461 communities (called groups in Sina Weibo platform) organized according to different interests. Because there are some special dimensions of data, e.g., community detail information (number of members, internal edges and external edges), related positive behaviors, and time slice labels, which must be included in our proposed D-CIEM for influence evaluation, the dataset in experiment is manually collected and all data dimensions in our experiments are labelled manually.

The details of our experiment are listed in Table 2.

6.1. Performance Simulation of Entity Influence in Social Networks. We first examine the performance of the proposed average intention degree of information propagation. To do this, we selected approximately 400 users at random to verify their intention of propagating information. We calculated their average intention degrees of information propagation and then recorded their information propagation-related behaviors (posting and forwarding) in the social network. We used the following factor for propagating ratios:

$$
\begin{gathered}
\text { propagation_ratio }\left(u_{i}\right) \\
=\frac{\mid \text { posting }\left(u_{i}\right)|+| \text { forwarding }\left(u_{i}\right) \mid}{\mid \text { total_behavior }\left(u_{i}\right) \mid}
\end{gathered}
$$

where $\mid$ posting $\left(u_{i}\right) \mid$ and $\mid$ forwarding $\left(u_{i}\right) \mid$ denote the total numbers of posting and forwarding behaviors of user $u_{i}$, while $\mid$ total_behavior $\left(u_{i}\right) \mid$ denotes the total number of behaviors of user $u_{i}$. We compared the performance of our proposed method with the method of single propagation 
ratio method $(S P R)$ using $\operatorname{Pro}\left(u_{i}\right) / A c S\left(u_{i}\right)$. As Figure 3(a) shows, the average value of propagation_ratio increases as the aip values increase. Most users (those whose aip factors are larger than 0.7 ) propagate information more actively than others. The average user influence degree increases as the aip values increase as shown in Figure 3(b). Obviously, we can see that the AIP proposed in this work aims to signify the inherent willing for information propagation, while the SPR only reveals the ratios of propagation based on historical data. The results of this examination verify that our proposed AIP has a better performance for estimate the probability of user information propagation than SPR, which also means that our proposed aip factor can be used to reflect the role of influence diffusion among social network users.

To show the performance of proposed entity influence in D-CIEM, we use the factor of influence spread in our experiment. When a user exhibits one or more activation behaviors (i.e., commenting, forwarding, approving, or labelling) in propagating information to other users, he or she is defined as an "activated user" in influence spread. Therefore, the factor of influence spread reveals the scope at which selected entities spread influence in the social network, including both users and communities. This factor is widely used in performance analysis of influence evaluation. For comparison purposes, we set the following groups for our examination:

(i) WUI: the top $k$ users based on user influence evaluation without dynamic perspective

(ii) DUI: the top $k$ users based on user influence evaluation in D-CIEM

(iii) HDUI: the top $k$ users based on the high degree evaluation method for user influence

(iv) DDUI: the top $k$ users based on the degree discount method for user influence

(v) RandomUI: $k$ users at random

(vi) WCI: the top $k$ communities based on community influence evaluation without dynamic perspective

(vii) DCI: the top $k$ communities based on community influence evaluation in D-CIEM

(viii) AVACI: the top $k$ communities based on the average value of user influence degrees in community

(ix) HDCI: the top $k$ communities based on the high degree method by treating the whole community as a single user

(x) DDCI: the top $k$ communities based on the degree discount method by treating the whole community as a single user

(xi) RandomCI: $k$ communities at random.

As shown in Figures 4(a)-4(b), the proposed entity influence groups (DUI and DCI) obtain the best performances. These results occur because (1) the influence degrees of DUI and DCI are the newest values: the older influence degrees are attenuated in D-CIEM and play less of a role in entity influence calculation; (2) the intention to propagate information is considered as a significant factor in entity influence evaluation, which plays an important role in influence spreading; (3) the user influence degree is calculated based on many additional factors, such as the in-degree and out-degree information, rather than only a summary of user past behavior; and (4) community influence evaluation considers more detailed community factors such as the number of members, relationship tightness degree, and structural instability, all of which are ignored in the other groups.

We examine the impact of the value of the attenuation coefficient $\omega$ to achieve a dynamic perspective. Because the attenuation coefficient has a major impact on dynamic influence evaluation, a reasonable value for this coefficient is essential in D-CIEM. We examine the performance of entity influence for groups of DUI and DCI. As shown in Figures $4(\mathrm{c})-4(\mathrm{~d})$, the empirical analysis leads to an optimal threshold value of $0.8-0.9$ for $\omega$.

\subsection{Performance Simulation of Community-Initiated} Influence. In this section, we verify the performance of community-initiated influence in D-CIEM. We use the activating ratio factor to verify whether a user or a community would be activated in a community's information propagation. First, we provide some justifications for the simulation settings:

(i) Information propagation from community: we define the information propagated by a community's internal users as information propagation from the community.

(ii) Activated user: if a user exhibits a positive response to information receipt during the propagation process, he or she is active in information propagation.

(iii) Activated community: when more than half of the activated users in a community are in another community's information propagation, the community is activated.

(iv) Community-initiated influence-based activation ratio: this factor denotes the ratio of the number of activated users within a certain influence value interval of community-initiated influence to the total number of users within the same influence value interval of community-initiated influence. This ratio can be one of four types: IIAR (influence of community on internal user-based activated ratio), IEAR (influence of community on external user-based activated ratio), INCAR (influence between nonoverlapping community-based activated ratio), and IOCAR (influence between overlapping community-based activated ratio).

(v) Community influence-based activating ratio $(C A R)$ : the CAR factor is the ratio of the number of activated users in an influence value interval of community influence to the total number of users in the same influence value interval of community influence.

For comparison, we record the above average values of activated ratios after 5,000 information propagation steps 


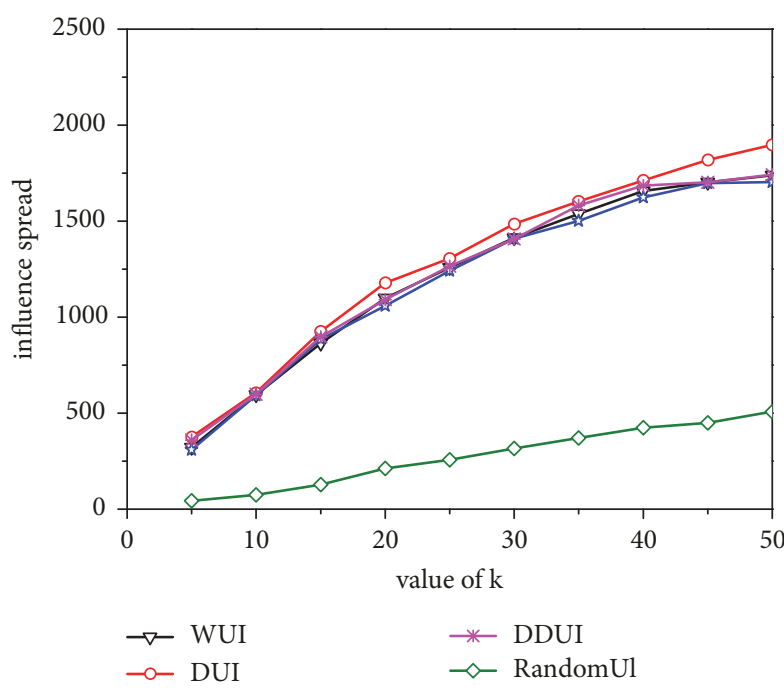

(a)

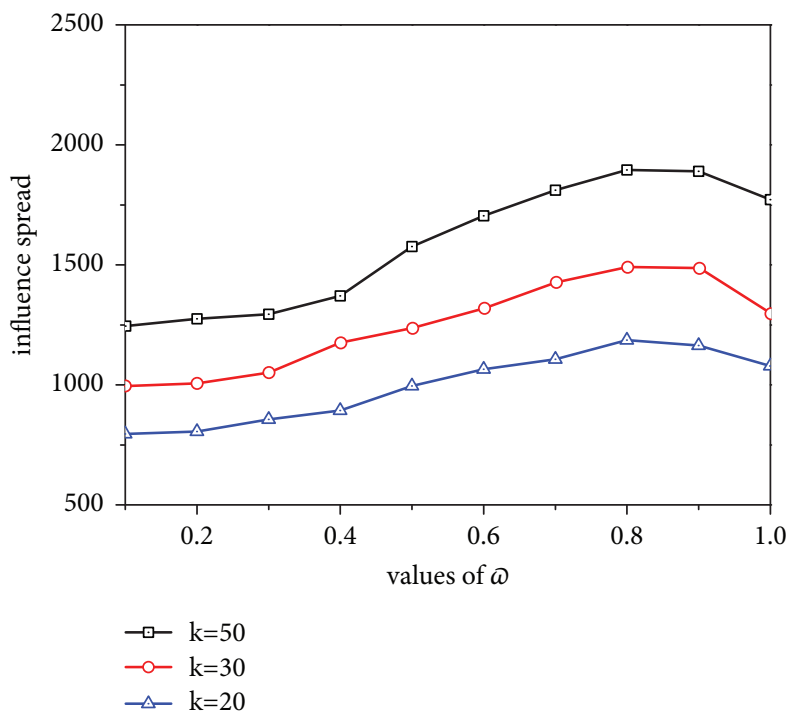

(c)

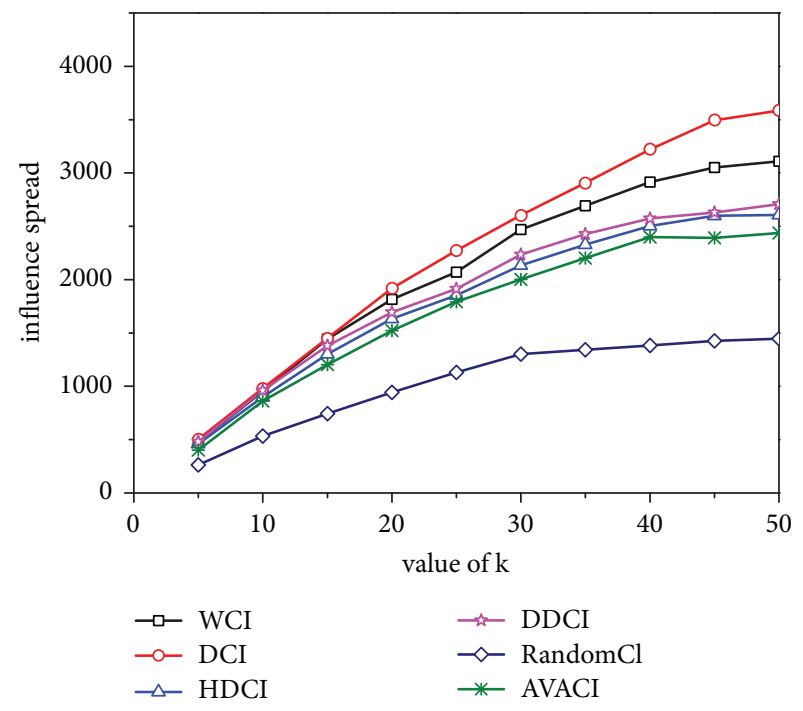

(b)

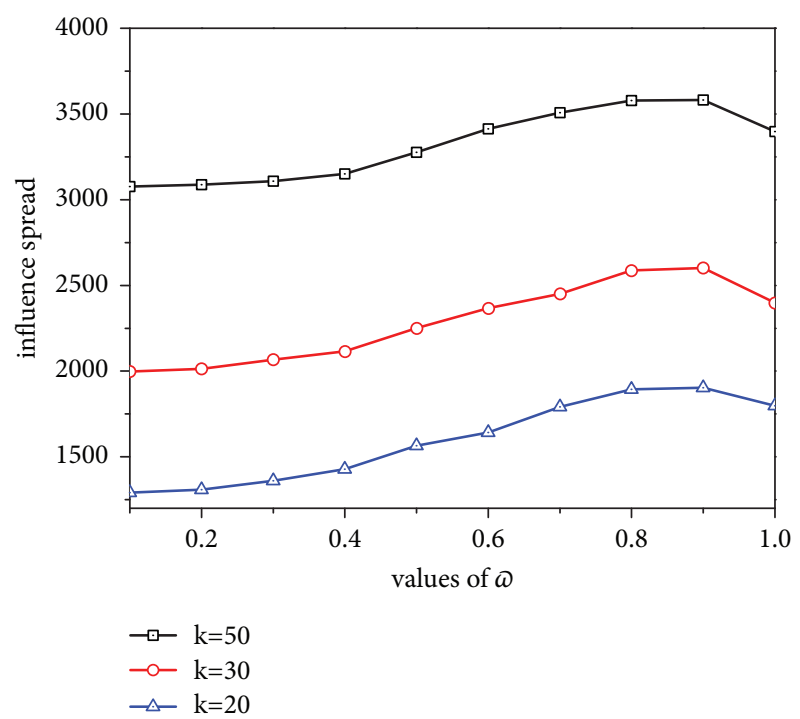

(d)

FIgURE 4: Performance analysis of the proposed entity influence.

to show the impacts of community-initiated influence on entities in the social network. The results are listed in Table 3.

We examine the effects of our proposed dynamic perspective on community-initiated influence. For comparison, we set four baselines that do not take dynamic perspective into account, notated as $N-I I A R, N-I E A R, N-I N C A R$, and $N$-IOCAR. We only compare the performances of groups with values larger than 0.7. The results are listed in Figures 5(a)-5(d). Our proposed community-initiated influence with dynamic perspective methods achieve better performances than the ones without dynamic perspective because the newest influence degrees contribute more in communityinitiated influence aggregation with dynamic perspective, resulting in more accurate influence degree evaluation.

Based on all the experimental results in this section, we can make the following conclusions: (1) as the value range increases, the values of IIAR and IEAR increase, demonstrating that a higher value of our proposed influence has greater impacts on both internal and external users. The results show that our proposed concepts of the influence of community on internal and external users are both feasible and effective in showing community influence on both its internal and external users. Most communities have more impact on their internal users than on their external users; (2) the impact of overlapping communities is larger than the impact of nonoverlapping communities. This occurs because overlapping communities involve internal users, resulting in a greater probability that over half of the users in the overlapping community will be activated; (3) a community has more influence on individual users than on other communities; and (4) our proposed community-initiated influence can more effectively show the impact of a community on other entities 


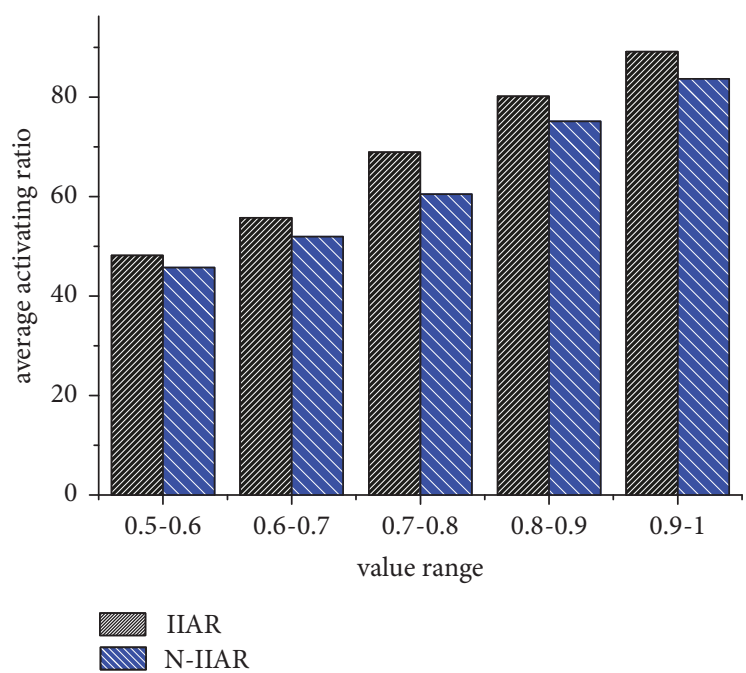

(a)

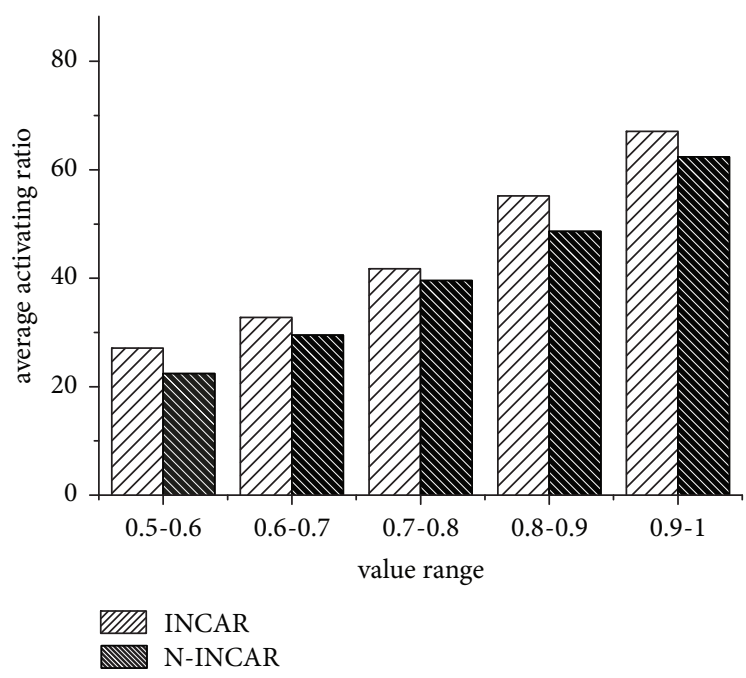

(c)

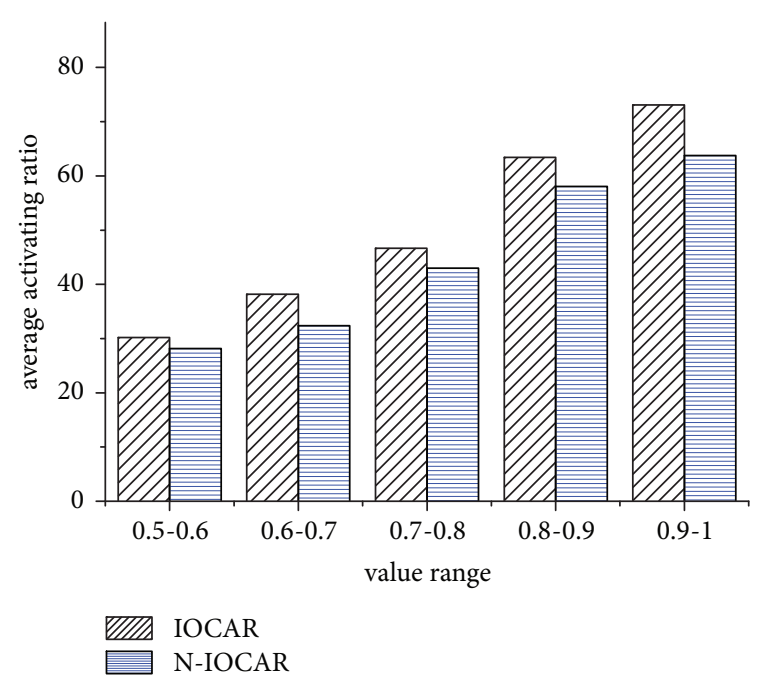

(b)

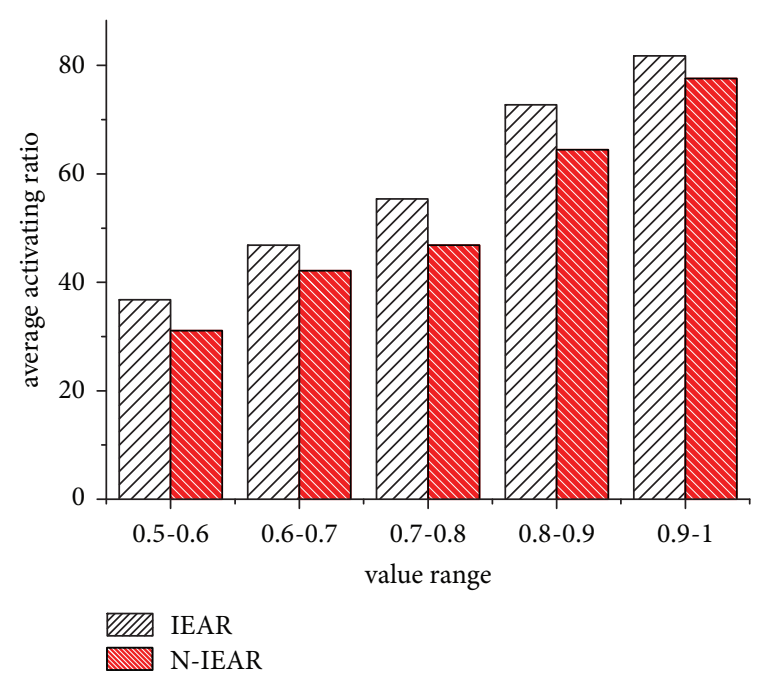

(d)

FIGURE 5: Performance comparison of the proposed community-initiated influence with dynamic perspective.

TABLE 3: Results of performance simulation of community-initiated influence.

\begin{tabular}{lccccc}
\hline Value range & IIAR & IEAR & INCAR & IOCAR & CAR \\
\hline $0-0.1$ & $6.82 \%$ & $3.87 \%$ & $0.76 \%$ & $1.31 \%$ & $3.71 \%$ \\
$0.1-0.2$ & $14.75 \%$ & $8.16 \%$ & $2.65 \%$ & $3.05 \%$ & $8.09 \%$ \\
$0.2-0.3$ & $22.92 \%$ & $13.87 \%$ & $6.97 \%$ & $7.59 \%$ & $12.85 \%$ \\
$0.3-0.4$ & $30.19 \%$ & $20.13 \%$ & $11.89 \%$ & $13.74 \%$ & $18.61 \%$ \\
$0.4-0.5$ & $39.89 \%$ & $27.51 \%$ & $19.37 \%$ & $21.94 \%$ & $25.65 \%$ \\
$0.5-0.6$ & $48.14 \%$ & $36.78 \%$ & $27.13 \%$ & $30.18 \%$ & $34.91 \%$ \\
$0.6-0.7$ & $55.71 \%$ & $46.83 \%$ & $32.78 \%$ & $38.17 \%$ & $42.73 \%$ \\
$0.7-0.8$ & $68.92 \%$ & $55.38 \%$ & $41.76 \%$ & $46.65 \%$ & $56.03 \%$ \\
$0.8-0.9$ & $80.15 \%$ & $72.71 \%$ & $55.18 \%$ & $63.41 \%$ & $69.17 \%$ \\
$0.9-1$ & $89.13 \%$ & $81.76 \%$ & $67.06 \%$ & $73.06 \%$ & $80.14 \%$ \\
\hline
\end{tabular}

than can community influence. Only a community with high entity influence would not always have large impacts on all its accessible users in a social network, while the proposed community-initiated influence is a more targeted approach that shows the community impact on its users. We also notice that there is a limitation in our experiment: only one database is used in this examination because of the requirements of data dimensions. And also, we will conduct our experiments on two more datasets in our future work.

\section{Conclusions}

In social networks, measuring entity influence is both common and inevitable. Community influence evaluation is the inner motivation behind a user's daily communications and interactions because users often have one or more identities in different communities. In this study, we propose a community influence evaluation scheme named D-CIEM that includes a dynamic perspective. Our work focuses on the following aspects: (1) a dynamic perspective for influence 
evaluation is introduced to consider influence attenuation effects as time passes; (2) entity influence, including both user and community influence, is calculated based on an information propagation intention evaluation; and (3) a community-initiated influence is presented to signify the binary degree of influence from a community to other entities in a social network.

The results justify the performance of our proposed DCIEM and show the effectiveness of our dataset. To conclude, the analysis of the experimental results shows that the results based on our proposed method are in line with the true results. The proposed D-CIEM can reveal community impact on both users and other communities. The ability to calculate such impacts can benefit many aspects of communities in social networks, for example, by enhancing the influence propagation process of a community, enlarging the sphere of community influence, and optimizing the ranking of a community in social networks. Our future work will examine our D-CIEM in more social network datasets and also focus on maximizing community influence while considering dynamic perspectives in social networks.

\section{Conflicts of Interest}

The authors declare that they have no conflicts of interest.

\section{Acknowledgments}

This work is supported by the National Natural Science Foundation of China (nos. 61572326, 71571136, and 61103069), Natural Science Foundation of Shanghai (18ZR1428300), the Shanghai Committee of Science and Technology (17070502800 and 16JC1403000), and the Innovation Program of Shanghai Municipal Education Commission (C160049).

\section{References}

[1] Z. Zhu, J. Su, and L. Kong, "Measuring influence in online social network based on the user-content bipartite graph," Computers in Human Behavior, vol. 52, pp. 184-189, 2015.

[2] F. Zhou, J. Jiao, and B. Lei, "A linear threshold-hurdle model for product adoption prediction incorporating social network effects," Information Sciences, vol. 307, pp. 95-109, 2015.

[3] G. Song, X. Zhou, Y. Wang, and K. Xie, "Influence maximization on large-scale mobile social network: a divide-and-conquer method," IEEE Transactions on Parallel and Distributed Systems, vol. 26, no. 5, pp. 1379-1392, 2015.

[4] J. C. Ji, X. X. Han, and Z. Wang, "Community influence maximizing based on comprehensive cascade diffuse model," Journal of Jilin University, vol. 47, no. 5, pp. 1032-1034, 2009.

[5] G. W. Park, S. H. Seo, S. J. Lee, and S. H. Lee, "Influencerank: trust-based influencers identification using social network analysis in Q\&A sites," IEICE Transactions on Information and Systems, vol. 95, no. 9, pp. 2343-2346, 2012.

[6] Y.-C. Chen, W.-C. Peng, and S.-Y. Lee, "Efficient algorithms for influence maximization in social networks," Knowledge and Information Systems, vol. 33, no. 3, pp. 577-601, 2012.
[7] N. Agarwal, H. Liu, L. Tang, and S. Y. Philip, "Modeling blogger influence in a community," Social Network Analysis \& Mining, vol. 2, no. 2, pp. 139-162, 2012.

[8] Y.-C. Chen, W.-Y. Zhu, W.-C. Peng, W.-C. Lee, and S.-Y. Lee, "CIM: community-based influence maximization in social networks," ACM Transactions on Intelligent Systems and Technology, vol. 5, no. 2, pp. 529-544, 2014.

[9] J. Li, W. Peng, T. Li, T. Sun, Q. Li, and J. Xu, "Social network user influence sense-making and dynamics prediction," Expert Systems with Applications, vol. 41, no. 11, pp. 5115-5124, 2014.

[10] U. Abelein and B. Paech, "Understanding the influence of user participation and involvement on system success-a systematic mapping study," Empirical Software Engineering, vol. 20, no. 1, pp. 28-81, 2013.

[11] S. E. Gibbs, L. Cu Le, H. B. Dao, and R. W. Blum, "Peer and community influences on the acceptance of premarital sex among Vietnamese adolescents," Journal of Paediatrics and Child Health, vol. 50, no. 6, pp. 438-443, 2014.

[12] H. Kim, K. Beznosov, and E. Yoneki, "A study on the influential neighbors to maximize information diffusion in online social networks," Computational Social Networks, vol. 2, no. 1, pp. 115, 2015.

[13] A. E. Cano, S. Mazumdar, and F. Ciravegna, "Social influence analysis in microblogging platforms-a topic-sensitive based approach," Semantic Web, vol. 5, no. 5, pp. 357-403, 2014.

[14] J. Zhou, Y. Zhang, and J. Cheng, "Preference-based mining of top-K influential nodes in social networks," Future Generation Computer Systems, vol. 31, no. 1, pp. 40-47, 2014.

[15] K. Lewis, M. Gonzalez, and J. Kaufman, "Social selection and peer influence in an online social network," Proceedings of the National Acadamy of Sciences of the United States of America, vol. 109, no. 1, pp. 68-72, 2012.

[16] N. Barbieri, F. Bonchi, and G. Manco, "Topic-aware social influence propagation models," Knowledge and Information Systems, vol. 37, no. 3, pp. 555-584, 2013.

[17] Y. Zhu, W. Wu, Y. Bi, L. Wu, Y. Jiang, and W. Xu, "Better approximation algorithms for influence maximization in online social networks," Journal of Combinatorial Optimization, vol. 30, no. 1, pp. 97-108, 2015.

[18] Y. Lin and J. C. S. Lui, "Analyzing competitive influence maximization problems with partial information: An approximation algorithmic framework," Performance Evaluation, vol. 91, pp. 187-204, 2015.

[19] Y. Qin, J. Ma, and S. Gao, "Efficient influence maximization under TSCM: a suitable diffusion model in online social networks," Soft Computing, vol. 21, no. 4, pp. 827-838, 2017.

[20] W.-X. Lu, C. Zhou, and J. Wu, "Big social network influence maximization via recursively estimating influence spread," Knowledge-Based Systems, vol. 113, pp. 143-154, 2016.

[21] S. Jendoubi, A. Martin, L. Liétard, H. Ben Hadji, and B. Ben Yaghlane, "Two evidential data based models for influence maximization in Twitter," Knowledge-Based Systems, vol. 121, pp. 58-70, 2017.

[22] T. Zhu, B. Wang, B. Wu, and C. Zhu, "Maximizing the spread of influence ranking in social networks," Information Sciences, vol. 278, pp. 535-544, 2014.

[23] L. Zhou and K. Lü, "Detecting communities with different sizes for social network analysis," The Computer Journal, vol. 58, no. 9, pp. 1894-1908, 2015.

[24] D. Wang, P. Wang, M. Li, and B. Zhang, "A topic space oriented user group discovering scheme in social network: a trust chain 
based interest measuring perspective," Scientific Programming, vol. 2016, pp. 1-25, 2016.

[25] A. Bozorgi, H. Haghighi, M. Sadegh Zahedi, and M. Rezvani, "INCIM: a community-based algorithm for influence maximization problem under the linear threshold model," Information Processing \& Management, vol. 52, no. 6, pp. 1188-1199, 2016.

[26] I. A. Christensen and S. Schiaffino, "Social influence in group recommender systems," Online Information Review, vol. 38, no. 4, pp. 524-542, 2014.

[27] B. Zhang, Q. Song, R. Peng, Z. Huang, and Q. Xu, "A novel community influence evaluation scheme based on information propagation in social network," International Journal of Computational Science and Mathematics, vol. 7, no. 1, pp. 29-41, 2016.

[28] Z. Bo, Z. Huan, L. Meizi, Z. Qin, and H. Jifeng, "Trust Traversal: A trust link detection scheme in social network," Computer Networks, vol. 120, pp. 105-125, 2017. 


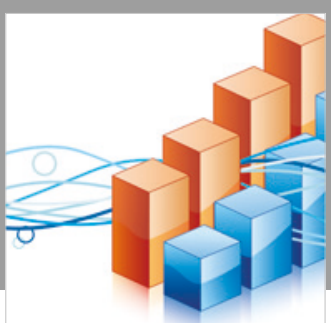

Advances in

Operations Research

\section{-n-m}
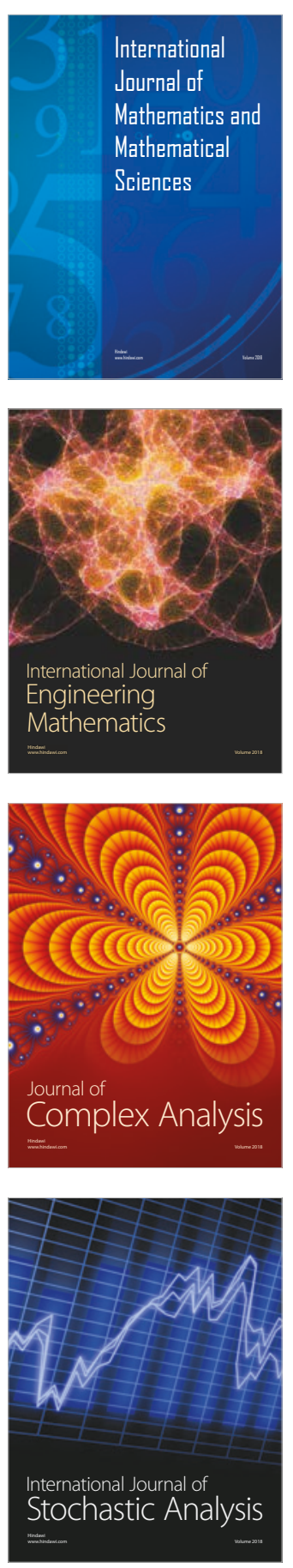
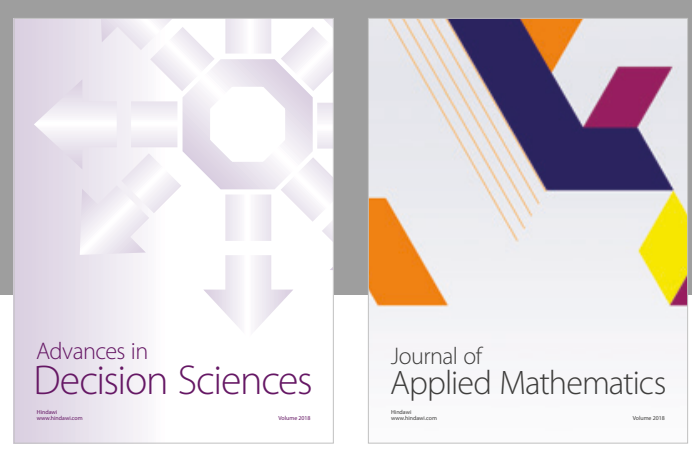

Journal of

Applied Mathematics
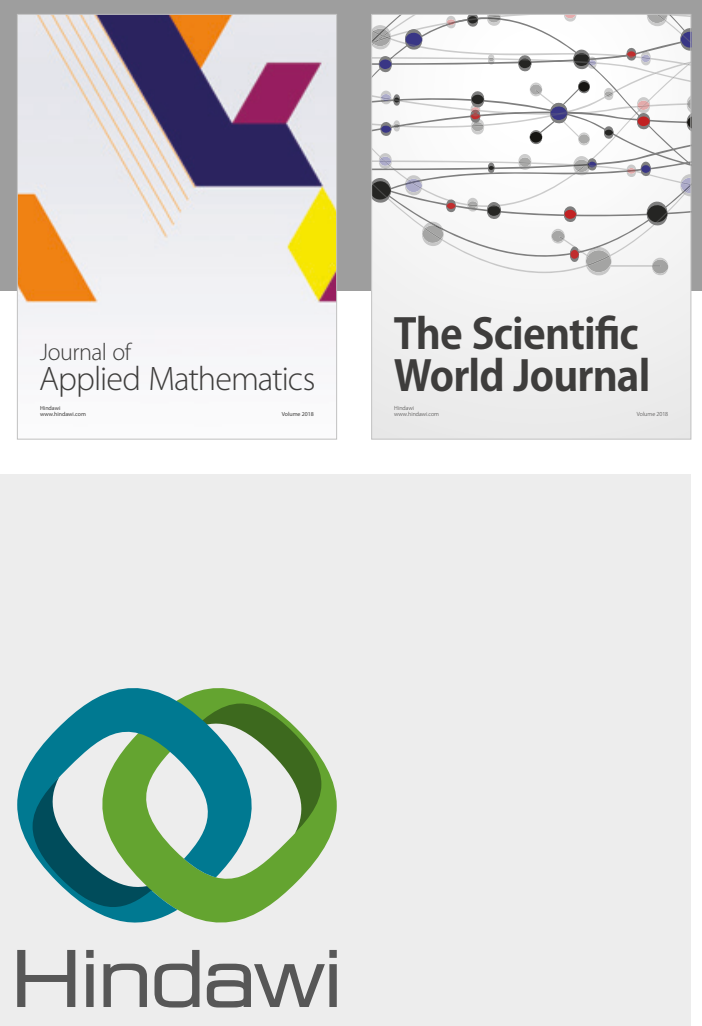

Submit your manuscripts at

www.hindawi.com

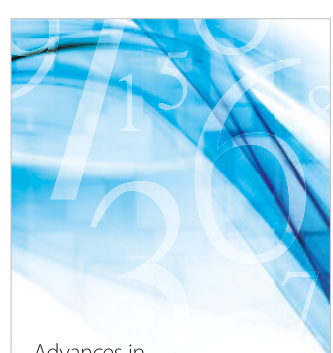

Advances in
Numerical Analysis
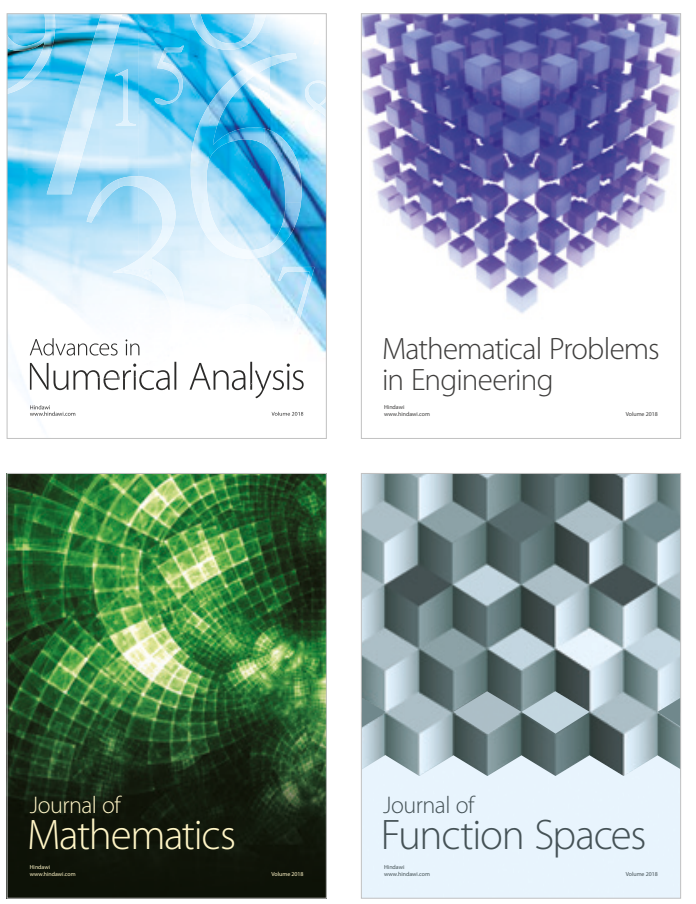

Mathematical Problems in Engineering

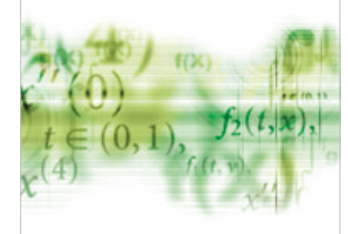

International Journal of

Differential Equations

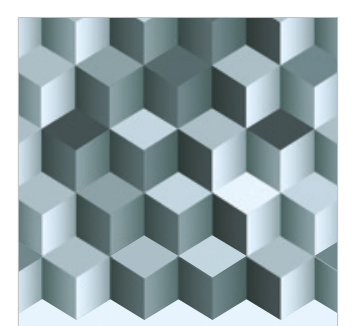

Journal of

Function Spaces

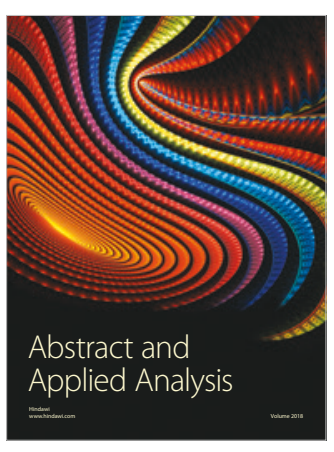

The Scientific

World Journal

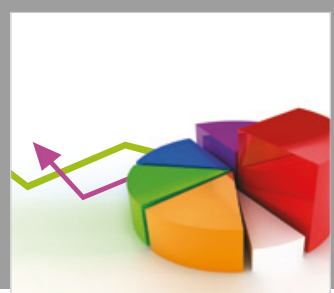

Journal of

Probability and Statistics
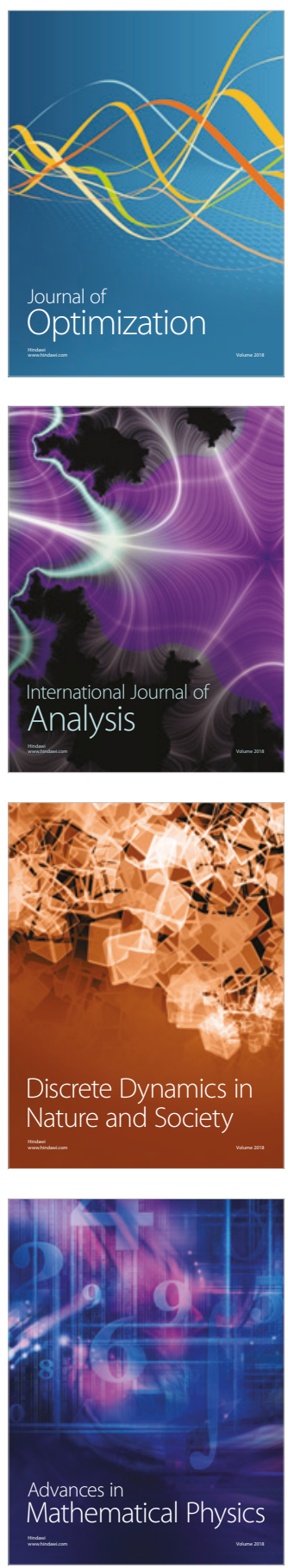